\title{
Beiträge zur Textkritik der Peschita.
}

\author{
Von Dr. Alfred Rahlfs.
}

Herr Prof. Gottheil hat kürzlich in den Mitteilungen des Akademisch-Orientalistischen Vereins No. 2 (Berlin 1889)

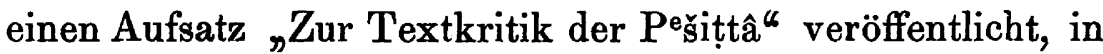
welchem er darauf aufmerksam macht, dafs der Text der Peschita, welchen Gregorius Bar Ebhraya in seinem Horreum mysteriorum commentiert, öfters von dem Texte abweicht, welcher uns gewöhnlich vorliegt. Um diese Thatsache festzustellen, hat er den von Bar Ebhraya ausgelegten Text der salomonischen Schriften mit dem Lee'schen Texte verglichen und die sich ergebenden Varianten zusammengestellt. Eine solche Collation, und zwar nicht blofs des Lee'schen Textes, sondern auch des codex Ambrosianus und der Urumiaer Ausgabe, habe ich natürlich selbst schon bei der Herausgabe der Anmerkungen des Bar Ebhraya zu den salomonischen Schriften angefertigt, aber sie nicht mit abdrucken lassen, da ich glaube, dafs zwar der künftige Herausgeber einer kritischen Peschitaausgabe das Horreum mysteriorum wird sorgfältig benützen müssen, dafs aber vorläufig die Zusammenstellung einzelner Varianten aus demselben ziemlich zwecklos ist. $\mathrm{Da}$ aber Herr Prof. Gottheil jene Collation einmal veröffentlicht hat, so sehe ich mich veranlalst, einige Nachträge und Berichtigungen zu derselben zu liefern. Zugleich benutze ich die Gelegenheit, um einige andere Untersuchungen zur Textkritik der Peschita wieder aufzunehmen, welche ich schon 1886 in Linden bei Hannover begonnen hatte, damals aber, da mir die nötigen Bücher nicht' zur Hand waren, liegen lassen mufste.

Die Siglen, welehe ich (im Anschlusse an Cornills Ezechiel) gebrauche, sind

Zeitschrift f. d. alttest. Wis8. Jahrgang 9. 1889. 
$a=$ codex Ambrosianus ed. Ceriani.

$\mathrm{e}=$ Psalter des Thomas Erpenius, nach seinem Tode Leyden 1625 herausgegeben.

$\mathbf{g}=$ Text des Gabriel Sionita, d. h. Text der Pariser Polyglotte, bei den Psalmen Text der 1625 in Paris erschienenen Ausgabe Gabriels. Ich unterscheide hiervon $\mathrm{p}=$ Pariser Polyglotte, $\mathrm{l}=$ Londoner Polyglotte, $l^{\prime}=$ Lee nur da, wo sie abweichen.

$\mathbf{u}=$ Bibel von Urumia.

Für die im 6. Bande der Londoner Polyglotte verglichenen Handschriften gebrauche ich die dort üblichen Bezeichnungen Poc., Uss. u. s. w.

Aufserdem habe ich folgende, der Berliner Königlichen Bibliothek gehörende Handschriften des syrischen Psalters benutzt :

$\alpha=$ Diez A. oct. 160, beendet im Tammuz 7007 Adams $=1499$ n. Chr.

$\beta=$ Diez A. quart. 118, beendet im Schebat 7015 Adams $=1507$ n. Chr.

$\gamma=$ Sachau 31, nach einer Bemerkung fol. $82^{1} 8.9$ geschrieben im Jahre $2038=1726 / 7$ n. Chr., am Anfang und Ende später (von einer anderen oder derselben Hand?) ergänzt.

$\delta=$ Sachau 225, beendet im Tischrin II des Jahres $2069=1757$ n. Chr.

$\varepsilon=$ Sachau 227, beendet im Schebat $2153=1842$ n. Chr.

$\zeta=$ Or. quart. 374 (im Anfange defect).

$\eta=$ Peterm. I 25 (im Anfange defect).

$\vartheta=$ Sachau 126 .

$\alpha \beta$ sind melchitische, $\gamma \delta \varepsilon \zeta \eta \vartheta$, so viel ich sehen kann, jakobitische Handschriften. 
I.

Der Hauptmangel der Collation Grottheils besteht darin, dafs Gottheil, da er einen Beitrag zur Textkritik der Peschita liefern wollte, blofs Lees Text zur Vergleichung mit dem Bibeltexte Bar Ebhrayas herangezogen hat. Samuel Lee hat zwar auch Handschriften benutzt (Classical Journal vol. 23, 245-249), aber, wie Cornill Ezechiel 139 f. nachweist, wesentlich doch nur einen Abdruck des Textes der Londoner Polyglotte geliefert, und diese wiederum bietet einen Abdruck aus der Pariser Polyglotte. Dals sich aber in einen solchen Abdruck eines Abdrucks neben den etwa angebrachten Verbesserungen auch Fehler einschleichen, ist a priori anzunehmen; als Beispiel führe ich Cant 8, 9 an, wo das richtige entstellt ist, und verweise auf das unten zu Cant 2, 8 Gesagte. Man mufs also die Pariser und Londoner Polyglotte zur Controlle heranziehen.

Weiter liegt uns seit einiger Zeit die von Ceriani herausgegebene Photolithographie des codex Ambrosianus vor, durch welche wir eine dem 6. oder 7. Jahrhundert entstammende Bibelhandschrift ganz genau kennen. Und selbst wenn Cornill mit seiner Behauptung (Ezechiel 145) Recht haben sollte, dafs unter allen zugänglichen Textesgestalten der Peschita a die schlechteste sei, so mufs doch jeder, der ex officio über Textkritik der Peschita schreibt, a immer vergleichen. Denn es wäre höchst sonderbar, wenn eine so alte Handschrift nicht wenigstens an einigen Stellen richtigere Lesarten haben sollte, als eine im Jahre 1824 erschienene Ausgabe, welche sich nur auf jüngere Handschriften stützt, grofsenteils nicht einmal direct auf Handschriften zurückgeht und keineswegs eine kritische Ausgabe genannt werden kann.

Endlich besitzen wir seit 1852 die Urumiaer Ausgabe des alten Testaments, welche auf nestorianischen Bibel- 
handschriften beruht. Es ist aus der Kirchengeschichte bekannt, dafs die Nestorianer des römischen Reiches, nachdem im Jahre 489 ihre Schule zu Edessa auf Befehl des Kaisers Zeno zerstört war, nach Persien auswanderten. Damals hatten die Christenverfolgungen in Persien aufgehört; die Sasaniden brauchten nicht mehr zu fürchten, dafs ihre christlichen Unterthanen mit den Christen des römischen Reiches sympathisieren würden, seit sie im Jahre 483 oder 484 auf der Synode zu Beth Lapat das nestorianische Glaubensbekenntnis angenommen und sich dadurch von jenen getrennt hatten $\left.{ }^{1}\right)$. Daher fanden die 489 aus dem römischen Reiche vertriebenen Nestorianer in Persien Schutz und Duldung. Sie grindeten eine neue Schule zu Nisibis, und die Gesamtheit der Nestorianer constituierte sich als eine besondere Religionsgemeinde mit eigenem Oberhaupte. So wurde der religiöse Gegensatz zwischen den Nestorianern und Monophysiten durch den politischen Gegensatz nur noch verschärft. Freilich änderte sich ja die politische Lage seit der Ausbreitung des Islam. Den fremden und andersgläubigen Eroberern gegenüber mochten sich die verschiedenen christlichen Parteien wieder mehr zusammenschliefsen, aber vereinigt haben sie sich nicht wieder. Die Jakobiten und Nestorianer haben immer ibre getrennte kirchliche Verfassung behalten.

Ich habe hieran kurz erinnern müssen, um die Bedeutung von $\mathfrak{u}$ für die Textkritik der Peschita klar zu legen. Was wir sonst erst mühsam suchen müssen, ist uns hier durch die Geschichte gegeben : die Handschriften

1) Nöldeke, Aufsätze zur persischen Geschichte 107. Die Quelle ist der Brief Simeons, Bischofs von Beth Arscham (ca. 510-525), über den Nestorianismus, bes. 10, $15 \mathrm{ff}$. des Abdrucks in Michaelis' Chrestomathie, sowie 13, $11 \mathrm{ff.} \mathrm{:}$ Darum haben wir uns von der Gemeinschaft der Nestorianer getrennt seit dem 27. Jahre des Königs Peroz [in welchem die Synode zu Beth Lapat gehalten wurde] bis auf den heutigen Tag und haben sie verdammt und verdammen sie und den Simon Magus, ihren ersten Lehrer u. s. w, 
der Peschita zerfallen in zwei Familien, in die nestorianischen und jakobitischen, oder in die östlichen und westlichen, wie sie Bar Ebhraya zu Psalm 10,5 unterscheidet. Dafs diese beiden Gruppen sich gegenseitig beeinflufst haben sollten, ist bei dem Gegensatze zwischen den Nestorianern und Jakobiten so gut wie ausgeschlossen. Wir können also durch Vergleichung der östlichen und westlichen Gruppe einen gemeinsyrischen Peschitatext herstellen, welcher mindestens so alt ist, wie die Trennung zwischen den Ostsyrern und Westsyrern, also wie das Jahr 484 oder 489.

Nun kennen wir zwar die beiden Gruppen nur unvollkommen; aber wir haben doch seit 1852 in u wenigstens Einen Vertreter der östlichen Gruppe und können seitdem vieles mit gröfserer Sicherheit entscheiden. Zwar wäre es möglich, dafs die amerikanischen Missionare, wie sie die in der Peschita fehlenden Stücke, z. B. die Perikope von der Ehebrecherin, eingesetzt haben, so auch andere Stellen, etwa nach dem Drucke Lees, geändert hätten. Aber da sie manche Lesarten bieten, welche von Bar Ebhraya als specifisch nestorianisch angegeben werden, so ist bis auf Weiteres anzunehmen, dafs sie einen im Ganzen treuen nestorianischen Text liefern.

Demnach sind für die Textkritik der Peschita folgende, allerdings bei der geringen Zahl und nicht absolut feststehenden Glaubwürdigkeit der Zeugen nicht ganz sichere Regeln aufzustellen :

1) Wo a g (westlich) und u (östlich) übereinstimmen, liegt ein bis in das letzte Viertel des 5. Jahrbunderts zurückreichender Text vor.

2) Wo a g gegen u übereinstimmen, ist eine besondere Untersuchung nötig, um zu constatieren, auf welcher Seite ein blofser Fehler oder eine absichtliche Correctur vorliegt.

3) Wo a u gegen g oder gu gegen a übereinstimmen, da ist es, obgleich Fälle vorkommen können, wo in zwei 
Handschriften unabhängig von einander derselbe Fehler gemacht ist, doch von vornherein wahrscheinlich, dafs die von einem östlichen und einem westlichen Zeugen beglaubigte Lesart älter ist, als die blofs von einem (event. auch von mehreren) westlichen beglaubigte.

Nach Mafsgabe der letzten Regel ergiebt sich z. B., was Gottheil allerdings aus der blofsen Vergleichung des von Bar Ebhraya benutzten Bibeltextes mit dem Texte Lees nicht lernen konnte, dafs die Lesarten Bar Ebhrayas

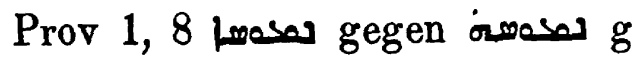

8, 22 gegen

13, 11 gegen gaso gave g

20, 17 متركا

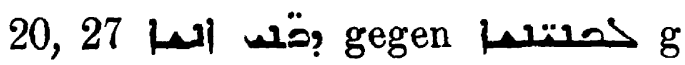

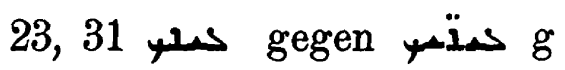

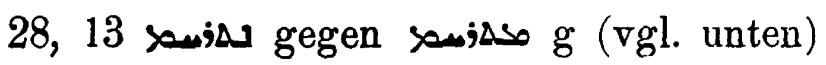

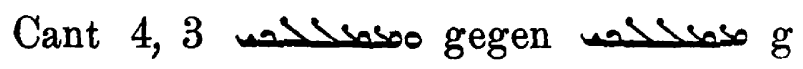

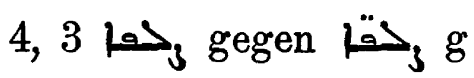

8, 9 : וli gegen $\mathrm{g}$

sämtlich alt und richtiger als die gs sind, weil sie sich ebenso in a $u$ finden. Hinzuzufügen sind noch folgende, von Gottheil nicht notierte Stellen :

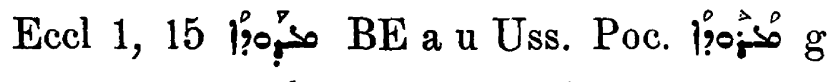

Cant 1, 8 BE a u

4,3 It BE a u LK (allerdings $\mathrm{LF}^{\circ}$ vocalisiert) $\mathrm{g}$.

An anderen Stellen stellt sich das Verhältnis der verschiedenen Zeugen zu einander anders. Doch ich beabsichtige nicht, alle von Gottheil zusammengestellten Varianten zu besprechen. Es genüge, die bei der Untersuchung einzuschlagende Methode dargelegt zu haben.

Ich gehe zu etwas Wichtigerem über : zu der Entdeckung Gottheils, dafs Bar Ebhraya einen Bibeltext be- 
nutzte, welcher an einer Anzahl von Stellen nach der ssyrischen Hexapla“ geändert war. Dals der Text der Peschita Aenderungen erlitten hat, wird wohl allgemein angenommen, wenn man auch im Einzelnen fast nichts darüber weifs. 'Vgl. zuletzt W. Wright, Syriac literature in Encycl. Brit. XXII 824 : „To what extent subsequent revision may have been carried it is not easy to say; but it seems tolerably certain that alterations were made from time to time with a view to harmonizing the Syriac text with that of the LXX ... On all these points, however, we know nothing for certain." Die Vermutung einer Correctur der Peschita nach dem Griechen wäre nunmehr, wenn Gottheil Recht hätte, wenigstens für Eine Handschrift zur Sicherheit erhoben. Es verlohnt sich also, die Stellen, an welchen Gottheil einen Einflus der LXX auf den Text Bar Ebhrayas entdeckt hat, genauer zu prüfen. Gottheil bemerkt :

1) Prov 5, 4 مكز- hat auch das so Syr.Hex." Dies

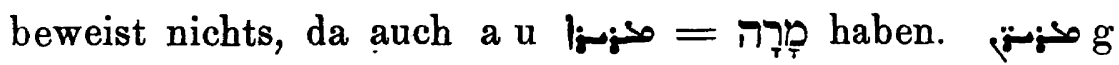
ist ein offenbarer grammatischer Fehler, da das Wort Prädicat $z u$ م ist.

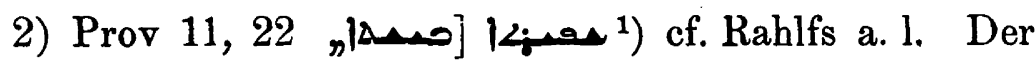
Fehler stammt aus der syr. Hex., welche schreibt [2al\} [so mufs es statt des bei Gottheil gedruckten heifsen]." Gottheil vergleicht hier die ᄁsyrische Hexapla“, aber er vergleicht sie unvollständig und kommt daher zu einer falschen Annahme. Die Peschita hat

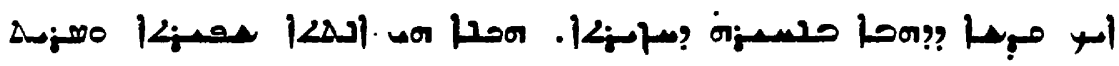

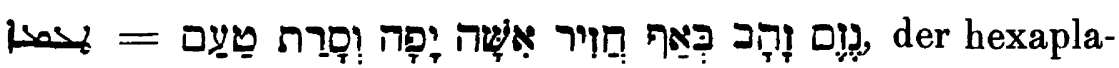

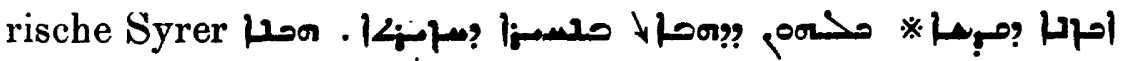

1) Die bei Gottheil zuerst stehende Lesart ist die Bar Ebhrayas, die andere die Lees. 


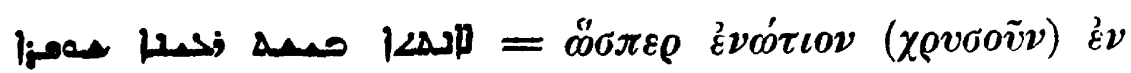

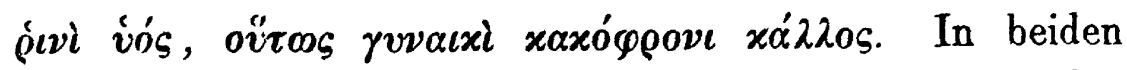
Fällen ist der Sinn derselbe, nur die Ausdrucksweise eine

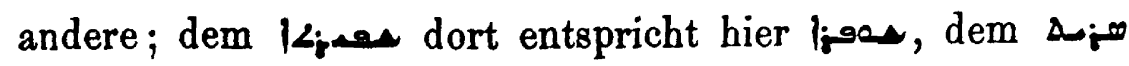

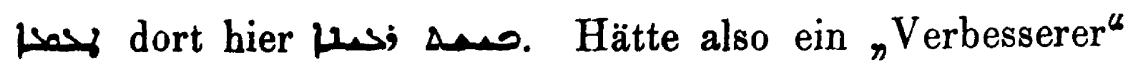
der Peschita statt or aus dem hexaplarischen Syrer das diesem entsprechende sul ; hätte das Sinn gehabt. Hätte er aber statt 1 ein diesem gar nicht entsprechendes مسيدj eingesetzt, so wäre das reiner Unsinn gewesen; er hätte die ganze Pointe der Stelle, welche in dem Gegensatze der Schönheit und Hälslichkeit liegt, verderbt. Für so dumm dürfen wir aber selbst einen Syrer kaum halten. Wir haben also nicht anzunehmen, dafs eine Lesart des Griechen von einem Corrector in den Text der Peschita eingesetzt wurde, sondern dafs der Peschitatext von einem Abschreiber geändert wurde. Diese Aenderung des Textes wird aber, woraut ich schon in einer Anmerkung meiner Ausgabe z. d. St. hingewiesen habe, erst den Abschreibern des Horreum mysteriorum zur Last fallen. Bar Ebhraya selbst las wie a ug |م wie aus seiner richtigen Erklärung „Der Thörin steht die Schönheit nicht an " mit Sicherheit folgt. Dals alle drei von mir benutzten Handschriften des $\mid$ i’ ; das falsche orieten, erklärt sich daraus, dafs sie auf Eine, mit dem Autographon Bar Ebhrayas nicht identische Handschrift zurückgehn (vgl. das Vorwort meiner Ausgabe IV). Der Schreiber dieser Handschrift machte sich eben bei dem langweiligen Geschäfte des Abschreibens seine eigenen Gedanken. Er sagte sich, ohne auf den ganzen Zusammenhang Acht zu geben: „Wie kann man von einer Frau in Einem Zuge aussagen, dals sie schön und häfslich sei? Offenbar mufs es nicht schön, sondern 
schlecht heifsen." Und voll Freude über seine Schlauheit brachte er diese Aenderung sofort in seinem Texte an, wie das ja auch unsere Setzer, nicht gerade zur Freude der Herausgeber, zuweilen thun sollen. Ich hätte also مen nicht blofs in die Anmerkung, sondern in den Text selbst aufnehmen müssen. Dafs ich es nicht gethan habe, ist ein Fehler, zu welchem mich übergrofse Vorsicht bei der Aufnahme von "Conjecturen" verleitet hat.

3) Prov 28, 13 saie. Die BE'sche Lesart scheint wieder aus der Syr. Hex. geflossen zu sein." Aber

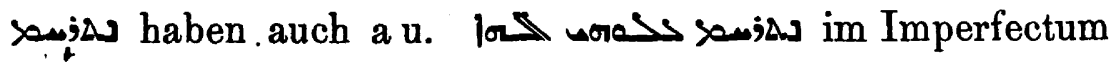
ist die correcte Uebersetzung des hebräischen Imperfects ם ירו: Das Participium in $\mathrm{g}$ scheint durch den Einflufs der vorhergehenden Participien entstanden zu sein.

4) Eccl 5, 1 ] Wort." Hier liegt ein offenbarer Irrtum Gottheils vor. Das Wort fehlt in $g$ nicht, sondern steht nur an anderer Stelle.

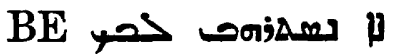

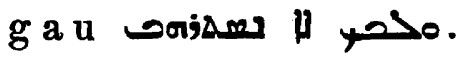

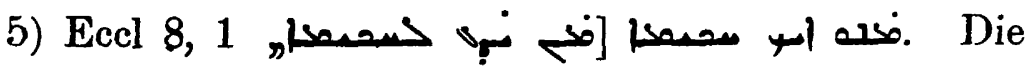
Lesart ist wieder aus der Syr. Hex. geflossen بطa معتصن." Dies ist der einzige Fall, in welchem eine Einwirkung seitens der LXX wahrscheinlich ist. $\mathrm{Zu}$ beachten ist übrigens, dals u ebenso wie Bar Ebhraya liest. Eine sichere Entscheidung lärst sich bei der Dürftigkeit des uns vorliegenden Materials noch nicht geben.

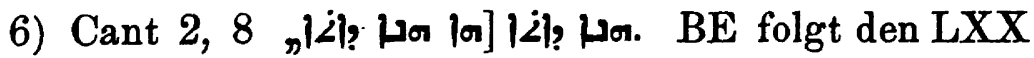

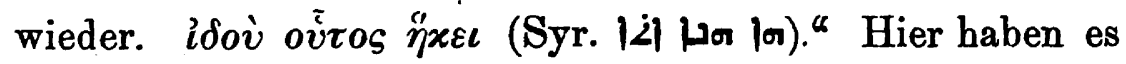
die Drucker der Londoner Polyglotte auf dem Gewissen, Gottheil auf eine falsche Fährte geführt zu haben. Die 


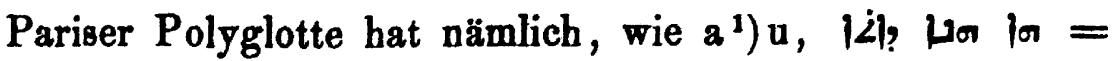
חָפר auch bei Lee ist lo vor dem ähnlich aussehenden tor ver-

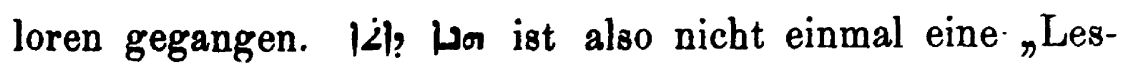
art", geschweige denn die ursprüngliche Lesart der Peschita, sondern nur ein Druckfehler.

7) Cant 2, 9 , lo] caret [.] LXX idoì oĩ Wieder ein Irrtum. Statt or lor $=7$ त $\mathrm{BE}$ a u Uss. hat $g$ lor or.

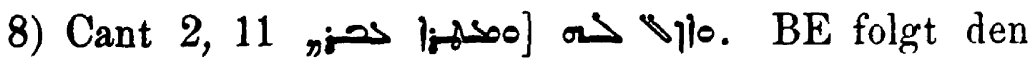

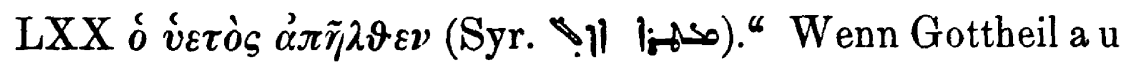
oder auch nur Poc. im 6. Bande der Londoner Polyglotte verglichen hätte, so würde er nicht als dem مهذم in $\mathrm{g}$ entsprechend o $\nabla$ \%lo angegeben haben. Jene bieten

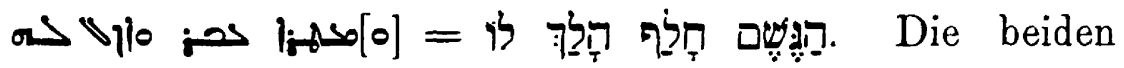
ersten Worte sind in g durch ó $\mu \circ \iota \tau \varepsilon \varepsilon \dot{\lambda} \varepsilon v \tau o v$ nach ausgefallen. Von einem Einflusse der LXX ist keine Spur zu entdecken.

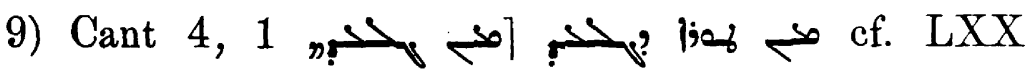

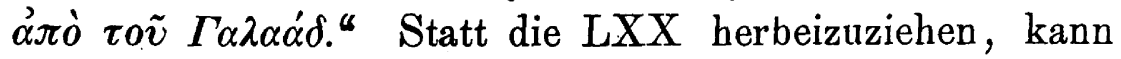
man die Auslassung von , las, das auch a u bieten, viel einfacher aus einer Nachlässigkeit Bar Ebhrayas oder des Schreibers der von ihm benutzten Bibelhandschrift erklären.

Wir sehen : unter den 9 Stellen, an welchen Gottheil einen Einfluls der LXX entdeckt hat, ist nur Eine (Eccl $8,1)$ übrig geblieben, an welcher ein solcher Einflufs wahrscheinlich, obgleich nicht sicher ist. Zweimal (No. 4 und 7) hat Gottheil sich geirrt. Viermal $(1,3,6,8)$ liegt

1) a hat jedoch $|2|$ statt $|2|$ ?. 
ein Schreib- oder Druckfehler bei g, resp. 1, einmal (9) eine Nachlässigkeit bei Bar Ebhraya, einmal (2) eine

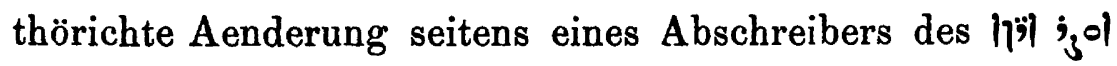
vor. Uebrigens. bätte Gottheil an manchen dieser Stellen gerade so gut behaupten können, der Text Bar Ebhrayas sei nach dem Hebräischen corrigiert, wie er es an Einer Stelle, Cant 1, 16, wirklich thut. Er schreibt :

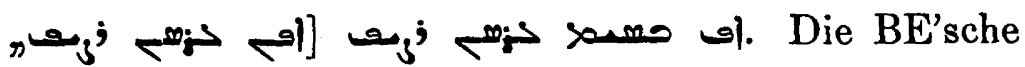
Lesart ist nach dem Hebr. corrigiert. Die Pešițtâ-Lesart

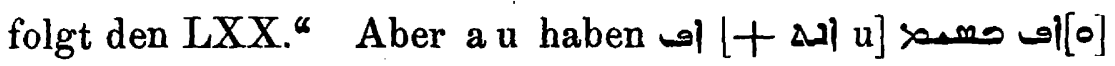

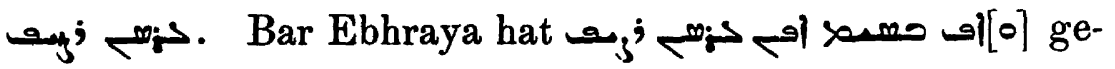
lesen, aber die beiden ersten Worte nicht mit commentiert. Das zweite 이 a $\mathbf{u}=$ ist, da schon ein 이 dicht vorhergeht, bei Bar Ebhraya zu of verderbt, bei g ganz ausgefallen.

Doch nicht zufrieden mit der Entdeckung dieser Correcturen auf Seiten Bar Ebhrayas, hat Gottheil, un auch Lee nicht ganz leer ausgehn zu lassen, auch auf seiner Seite eine Correctur nach LXX aufgespürt, aber mit nicht

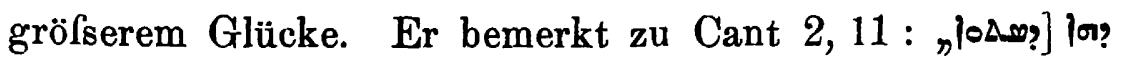

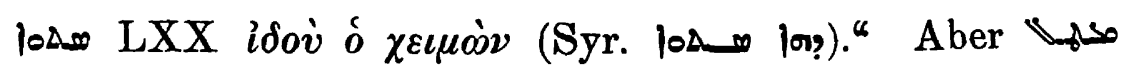
i jos lor, was auch a u bieten, ist die genaue Ueber-

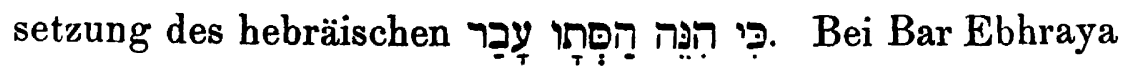
ist in ausgefallen.

Damit sind diese Entdeckungen Gottheils abgethan.

II.

Was Gottheil in den salomonischen Schriften irrtümlich sucht, findet sich im Psalter, freilich in anderer Weise. Nicht der von Bar Ebhraya benutzte Text des Psalters ist corrigiert, sondern erst Bar Ebhrayas Psalmencommen- 
tar hat den Anlafs zu einer Revision des syrischen Psalters gegeben.

Es ist bekannt, dafs Bar Ebhraya nicht viel von der Peschita gehalten hat, dafs er sie nur deshalb seinem Bibelcommentare zu Grunde gelegt hat, weil sie „überall in den Händen der Syrer war." Da er so urteilte, hat er sehr oft neben dem Texte der Peschita auch den der LXX, des Aquila, Symmachus, Theodotion u. a. angeführt und in manchen Fällen letzteren den Vorzug gegeben. Besonders zahlreich sind diese Anführungen aus den anderen Uebersetzungen in dem Commentare zu dem in der Kirche am meisten gebrauchten Buche des alten Testamentes, dem Psalter, und dies ist der Hauptgrund, weshalb P. de Lagarde diesen Teil des $|\not| j_{3}, 0 \mid$ in seinen Praetermissorum libri duo 1879 herausgegeben hat. Gerade hier finden sich auch manche Stellen, an welchen Bar Ebhraya den Text der Peschita für falsch erklärt und eine andere Lesart vorschlägt. Auf diese Stellen kommt es hier an. Ich stelle sie der Reihe nach zusammen, indem ich zugleich die entsprechenden Lesarten der Drucke a egu und der oben aufgeführten Berliner Handschriften angebe $^{1}$ ).

Bar Ebhraya bemerkt

1) zu Psalm 4, 5 |مخحتصف, . . nach der grammati-

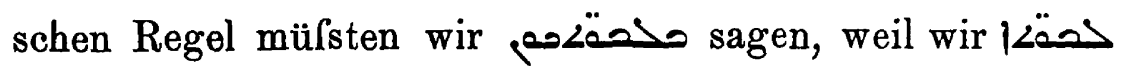
sagen und nicht weisen für die Ungebildetheit der Peschita."

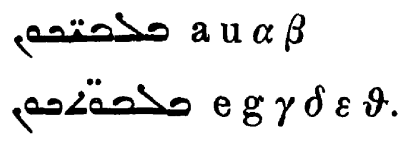

1) Wie ich nachträglich gesehen habe, hat schon Baethgen in seinen "Untersuchungen über die Psalmen nach der Peschita" Kiel 1878 , S. $20-22$ und in Jahrb. f. prot. Theol. 1882, S. 423 viele dieser Varianten angeführt, ohne jedoch ihre Bedeutung zu erkennen. 


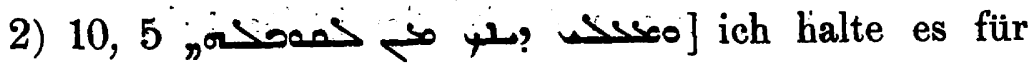
recht, dafs es mit zwei Lamadh geschrieben und مصحخم gelesen werde, d. h. owoj; 0 , weil auch der Grieche ow sagt; aber in allen östlichen und westlichen Handschriften, welche wir gesehen haben, ist es mit Einem Lamadh geschrieben."

क a e $u \beta \beta$ (in e findet sich der Druckfehler.

跨 $\mathrm{g} \gamma \delta \varepsilon \vartheta$.

3) 12,6 (0) mit Qof, und mit Recht; der Grieche إسم صدزملا, der

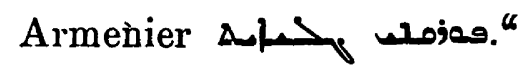

صم:م a g u $\alpha \beta \gamma \delta \varepsilon$ (in $\vartheta$ fehlt die Stelle).

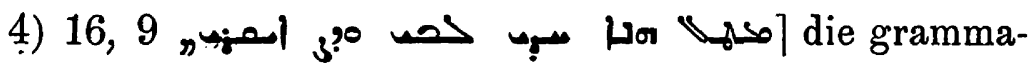

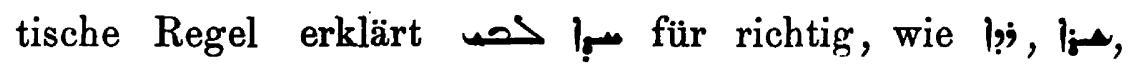

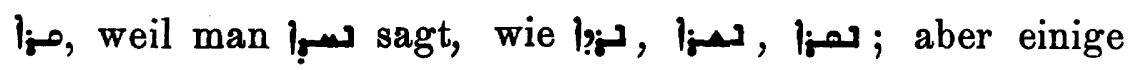
setzen טب: under eine besondere Regel. Der

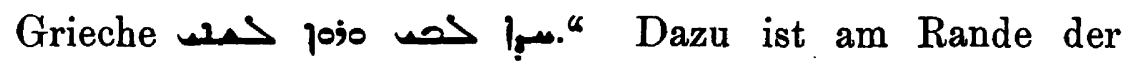

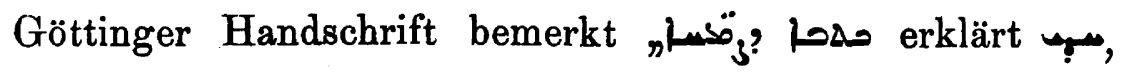
(Vür richtig." Martin, Oeurres grammaticales d' Abou'lfaradj I 105, 22 f.)

a e g u $\alpha \beta \gamma \delta \varepsilon \vartheta$.

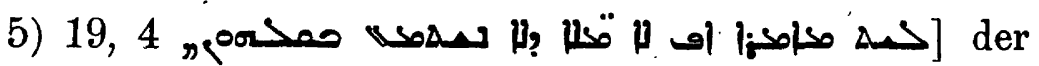

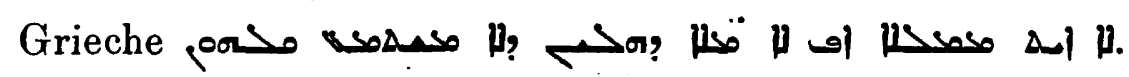
Der Sinn des Syrers ist hier dem des Griechen entgegengesetzt. Denn der Syrer sagt, dafs alle Reden mit ihrer Stimme gehört werden, und der Grieche, dafs ihre Stimme durchaus nicht gehört wird, und dies ist richtig, weil es die Absicht des Psalmisten ist zu zeigen, dafs sie schweigend predigen." 
a a e $\alpha \beta$

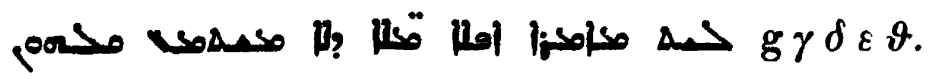

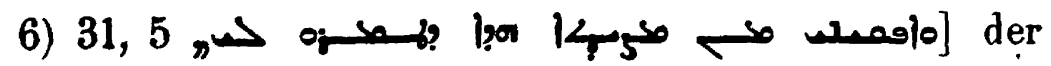

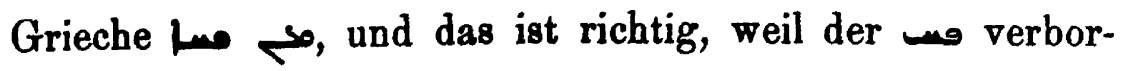
gen wird, aber nicht die 1 -

lian 1 a e u $\alpha \beta$

Ha tw

7) 33, 8 d. h. die Bewohner der Erde. Der Grieche und das ist richtig."

a a e u $\alpha \beta \gamma \delta \varepsilon \zeta \vartheta$

2 $\mathrm{g}$.

8) 35, 20 |م[ohne $\|$ ], und das ist richtig, weil der, welcher mit den Augen zwinkt, Friede sagt und Zorn denkt."

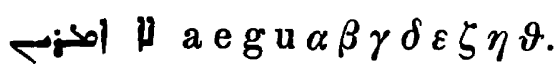

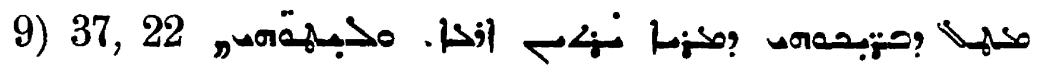

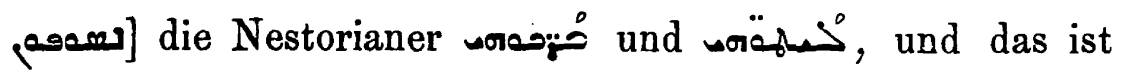

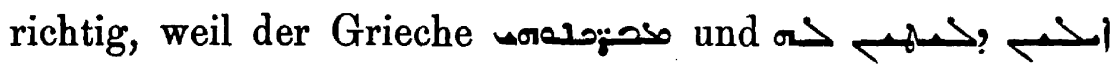
sagt."

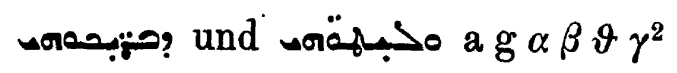

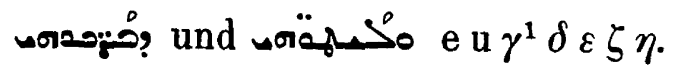

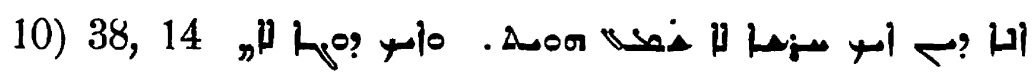

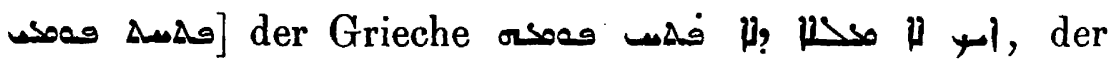

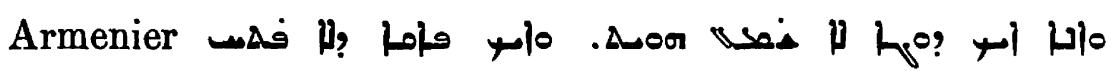
ob, und das ist richtig, weil der $7^{0}$ ? nicht hört, und der مام nicht spricht; denn der مزن kann hören, und der yoe kann sprechen." 


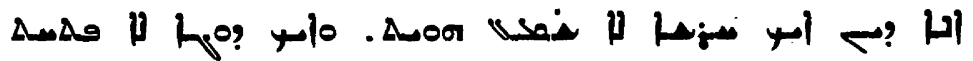
טمu $a \alpha \beta$

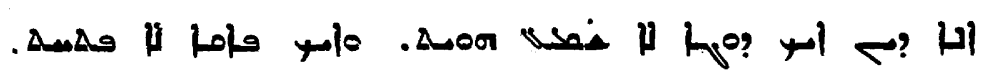
ט

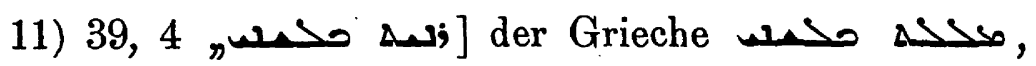
und dies ist richtig, weil das Denken mit dem Verstande und nicht mit der Zunge geschieht."

;

os eg $\gamma \delta \varepsilon \zeta \eta \vartheta$.

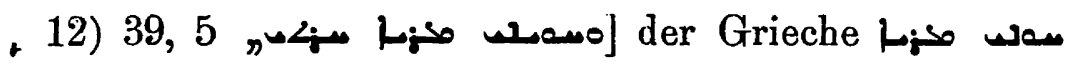
إو > sagt, und nicht مبر

weno a u $\alpha \beta$

we $\operatorname{eg} \delta \varepsilon \zeta \eta \vartheta$.

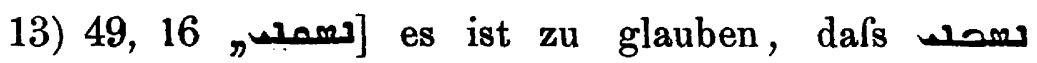
[gemeint] war, aber von dem ersten Schreiber mit Qof vertauscht wurde, weil der Grieche נهم > sagt, und der Armenier sc"

a e $\alpha \beta$

נח $\mathrm{g} \gamma \delta \varepsilon \zeta \eta \vartheta$.

14) 55, 21 [ن] dieser Stichos mufste im

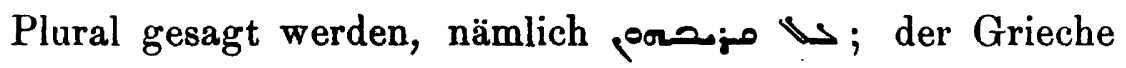

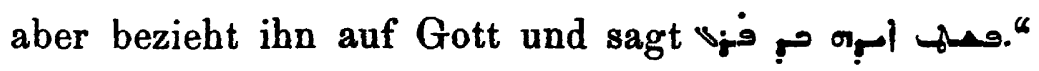

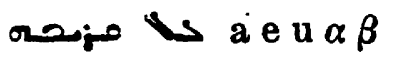

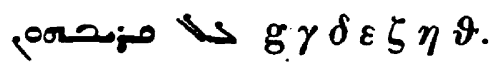

15) 72,9 , Handschriften ist so geschrieben, aber بحسي mit hartem Kaf vor Heth ist richtig." 


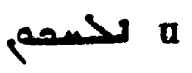

حصف

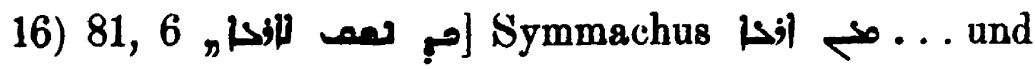
das ist richtig."

$\Delta ; \beta$ a e $\alpha \beta$

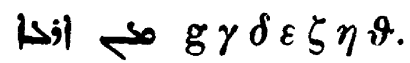

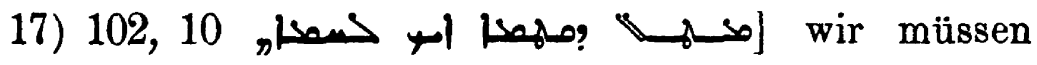
sagen."

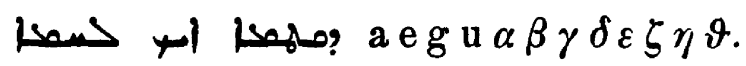

An diesen 17 Stellen handelt es sich um Aenderung von Consonanten. Diejenigen Stellen, an welchen es sich blofs um Vocale handelt, wie 8, 5. 26, 7. 35, 17, habe ich nicht mit aufgeführt.

Von diesen 17 Stellen sind noch einige auszuscheiden :

1) 12,6 wird a nar ein Schreibfehler des dem

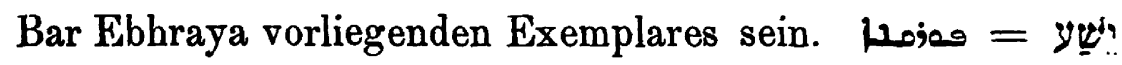
ist die richtige, von allen uns vorliegenden Zeugen gebotene Lesart.

2) 16, 9 ist in keiner Handschrift in مب geändert. Vielleicht hatte der Corrector ein Exemplar des $\left|1 ; i_{3} ; 0\right|$ vor sich, in welchem, wie in der Göttinger Handschrift, am Rande das anders lautende Urteil Bar Ebhrayas in مصا der Grammatik Bar Ebhrayas im Kopfe. Jedenfalls richtete er sich hier nicht nach dem Commentare, in welchem die Frage nur beiläufig, sondern nach der Grammatik, in welcher sie ex officio behandelt wird. Ein umgekehrter Fall liegt 102, 9 vor. Hier bemerkt Bar Ebhraya zu ס Deide Formen sind richtig wie خد ua in der Erzählungsform." Am Rande der Petermannschen Handschrift ist dazu bemerkt: 


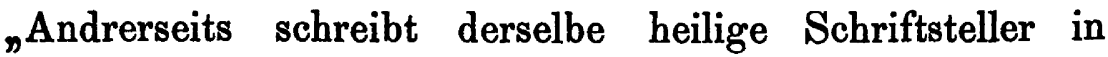
معبه u. s. w. zu lesen. Betet für mich [näml. für den Schreiber dieser Randnote]!“ (Vgl. Martin, Oeurres grammaticales I 127, 19 ff.) Hier hat nicht nur das nestörianische $u$ معه , sondern auch e g $\delta \varepsilon \zeta$ $\eta \vartheta$, während a $\alpha \beta \gamma$ مص山 haben. Hier ist also in jenen corrigiert; wieder richtete sich der Corrector nicht nach dem Commentare, sondern nach der Grammatik.

3) 72, 9 findët sich das (لحصa, welches Bar Ebhraya in keiner alten Handschrift gefunden hat, auch in der alten jakobitischen a und in den melchitischen $\alpha \beta$. Es ist also zweifelhaft, ob dies (كمس in $\gamma \delta \varepsilon \zeta \eta \vartheta$ aus vorgregorianischer Ueberlieferung oder nachgregorianischer Correctur stammt.

4) 35, 20 und 5) 102, 10 sind nicht geändert.

6) 33, 8 hat nur g بحص:2; dies könnte vielleicht aus Gabriel Sionitas eigener Weisheit hervorgegangen sein.

Aus den übrigen 11 Fällen (No. 1, 2, 5, 6, 9-14, 16) geht dagegen mit Sicherheit hervor, dafs

a $u \alpha \beta$ einen nicht corrigierten Text bieten, was übrigens von vornherein anzunehmen war, da a aus dem 6 . oder 7. Jahrhundert, $\mathrm{u}$ nestorianisch, $\alpha \beta$ melchitisch sind,

g $\gamma \delta \varepsilon \zeta \eta \vartheta$ nach Bar Ebhrayas $\left|\eta \eta^{\prime} ; 0\right|$ corrigiert sind, e einen gemischten Text aufweist.

Die Uebereinstimmung zwischen $\mathrm{g} \gamma \delta \varepsilon \zeta \eta \vartheta$ ist, von einigen Kleinigkeiten abgesehen, sowohl hinsichtlich des Corrigierten, als des nicht Corrigierten so grofs, dals ein zufälliges Zusammentreffen mir ausgeschlossen scheint. Ich nehme daher an, dafs nicht ein jeder auf eigene Faust corrigiert hat, was er für der Correctur bedürftig hielt, sondern dafs vor etwa $600 \mathrm{Jahren}$ von den Jakobiten eine officielle Revision des Psalters vorgenommen wurde. Dafs sie officiell war, schliefse ich daraus, dafs nicht einzelne,

Zeitschrift f. d. alttest. Wiss. Jahrgang 9. 1889. 
sondern alle jüngeren jakobitischen Handschriften, welche ich verglichen habe, jene Correcturen aufweisen. Von Gregorius Bar Ebhraya selbst wird diese Revision schwerlich herrühren; er würde wohl alle 17 Stellen corrigiert haben. Wir werden sie also seinen Schülern verdanken, welche von ihm gelernt hatten, wie „ungebildet ${ }^{*}$ die Peschita sei, und welche wenigstens in dem täglich gebrauchten Psalter das Gröbste nach der Anleitung ihres Meisters verbessern wollten. Für die Geschichte der syrischen Kirche beweist dies von neuem, ein wie nachhaltiger Einflufs auf ihre spätere Entwicklung, wenn man von einer solchen überhaupt sprechen kann, von .dem Maphrian" ausgegangen ist.

Gabriel Sionita hat nach seiner eigenen Angabe drei Handschriften der Psalmen benutzt.: eine nestorianische, eine jakobitische und eine maronitische. ,Wie sich aus dem Obigen ergiebt, ist er der jakobitischen Handschrift gefolgt; denn dafs die Revision der Jakobiten auch die maronitischen Handschriften beeinflufst haben sollte, ist unwahrscheinlich. Dafs die anderen Handschriften von dieser abwichen, lehrt Sionitas Variantenverzeichnis. Allerdings ist dieses sehr unvollständig und giebt die Varianten meistens blofs in lateinischer Uebersetzung. Aber wir wissen genug, wenn wir erfahren, dafs , in alio codice

$39, . .4$ meditatus sum statt locutus sum

39, 5 et ostendit mihi Dominus statt ostende mihi Domine

49, 16 extrahet me statt accipiet me zu finden ist.

Des Thomas Erpenius Psalmen sind gedruckt „ex duobus antiquissimis codicibus manuscriptis“. Ceriani, le edizioni e i manoscritti etc. 7 bemerkt dazu, dies sei eine „frase molto elastica per quel tempo". Dafs er Recht hat, folgt aus der obigen Tabelle. $e$ wechselt zwischen corrigierten und nicht corrigierten Lesarten, also bot die eine 
Handsehrift des Erpenius den revidierten T'ext, war also im Jahre 1625 schwerlich älter als 300 Jahre. Die andere Handschrift mochte älter sein. Erpenius verfuhr eklektisch. Ein Variantenverzeichnis giebt er nicht.

Für den 6. Band der Londoner Palyglotte sind drei Psalterien collationiert : eins stammte von Ussher, zwei von Pococke.

Poc. 1 ist, da Thorndike keine Varianten desselben zu den betreffenden Stellen angiebt, eine mit $g$ übereinstimmende, also jünge, jakobitische Handschrift.

Poc. 2 liest 102, امطعه alst, ist also westsyrisch, aber nicht corrigiert, denn es finden sich in ihm

$$
\begin{aligned}
& \text { 31, } 5 \text { ك- } 5 \\
& \text { 55, } 21 \text { منحسها } \\
& \text { 81, } 6 \text { p. }
\end{aligned}
$$

Dafs zu den übrigen Stellen keine Varianten aus Poc. 2 angeführt werden, ist aus der Ungenauigkeit der Arbeit Thorndikes zu erklären, welche Samuel Lee für die ersten acht Kapitel der Genesis durch eine Nachcollationierung bewiesen hat (Classical Journal vol. 23, 249, übersetzt und verbessert in Winer und Engelhardt, Neues krit. Journal der theol. Literatur I 159-161).

Uss. hat nach Thorndike im Texte

$$
\begin{aligned}
& \text { قמر 31, } 5 \\
& \text { 55, } 21 \text { منصسم } \\
& \text { 81, } 6 \text { | }
\end{aligned}
$$

aber die alten Lesarten sind am Rande notiert. Diese Randnoten kommen indessen nicht für uns in Betracht, da sie, wie Thorndike gefunden hat, aus der erpenianischen Ausgabe excerpiert sind (\$. 1 des syrischen Variantenverzeichnisses). Demnach ist auch Uss. corrigiert. Hiergegen beweișt nicht, dafs Uss. 102, 9 طعبه hat, da sich dies auch in dem corrigierten $\gamma$ findet. Wohl aber steht 
damit im Widerspruch, dafs nach Thorndikes Angabe Uss.

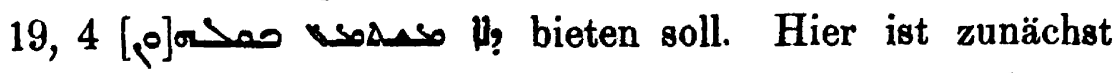
so gut wie sicher, dafs Thorndike die Lesart falsch angegeben hat. Die beiden vorkommenden Lesarten sind (1); letztere hat der Text der Polyglotte, also ist im Variantenverzeichnisse erstere zu erwarten. Wahrscheinlich ist mir aber ferner, dafs Thorndike sich auch in der Angabe der Handschrift geirrt und Uss. mit Poc. 2 verwechselt hat. Denn da Poc. 2, wie nachgewiesen, nicht corrigiert ist, so mufs sich

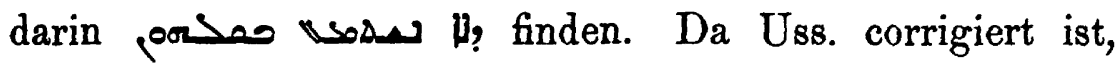
so ist in ihm keine Abweichung von dem Texte der Polyglotte zu vermuten. Die Annahme einer Verwechselung von Uss. und Poc. 2 würde alle Schwierigkeiten heben. Eine Berechtigung zu dieser Annahme wird man mir nicht absprechen können, da es constatiert ist, dafs Thorndike flüchtig gearbeitet hat.

Somit sind zu g [genauer : derjenigen Handschrift, welcher Gabriel Sionita gefolgt ist] $\gamma \delta \varepsilon \zeta \eta \vartheta$ noch drei corrigierte Psalterien hinzugekommen : eine Handschrift des Erpenius, Uss. und Poc. 1. Man sieht daraus, wie zahlreich diese Sorte von Handschriften ist. Man sieht aber weiter, wie alt die Handschriften waren, auf welche g e l basiert sind, und was wir von dem kritischen Werte dieser Ausgaben $\mathbf{z u}$ halten haben. Und endlich drängt sich die Frage auf : Wenn noch am Ende des 13. Jahrhunderts oder später eine solche Revision des heiligen Textes vorkommen konnte, sollte dann nicht auch in den früheren Zeiten Aehnliches passiert sein ?

\section{III.}

Die einzige Handschrift der Peschita, welche wir ganz genau kennen, ist die von Ceriani in Photolithographie herausgegebene, aus dem 6. oder 7. Jahrhundert stammende 
Handschrift der ambrosianischen Bibliothek in Mailand. Es ist daher für den augenblicklichen Stand der Peschitaforschung besonders wichtig, die Beschaffenheit des Textes dieser Handschrift durch eine gründliche Untersuchung festzustellen. Den 'Weg dazu hat Cornill, Ezechiel 140 -145 angebahnt, indem er den von a gebotenen Text des Ezechiel mit g $1 l^{\prime}$ sorgfältig collationiert und die sich ergebenden Varianten zusammengestellt hat. Er kommt zu dem Resultate : „Unter allen zugänglichen Textesgestalten der Peschito ${ }^{1}$ ) ist a die schlechteste : sie steht an Werth sogar noch tief unter Lee, und von diesem Gesichțpunkte aus ist das auf die Herausgabe von a verwendete Geld zum Fenster hinausgeworfen." Dieser kühn hingeworfene Satz, welchen Cornill auch auf der Generalversammlung der Deutschen Morgenländischen Gesellschaft in Karlsruhe, Sept. 1882, vorgetragen hat, frappierte mich gleich beim ersten Male, wo ich ihn las, derartig, dafs ich beschlofs, die denselben stützenden Gründe zu untersuchen. Diese Untersuchung hat nun ein etwas anderes Resultat ergeben, welches ich im Folgenden darlegen werde, indem ich Cornills Argumente der Reihe nach durchgehe.

Cornill beginnt, nachdem er die sich findenden Varianten zusammengestellt hat, seine Argumentation folgendermafsen : „Es mufs auf den ersten Blick befremden, dafs unter den 195 vorstehend mitgetheilten Varianten as sich eine auffallend grofse Zahl von Berührungen mit Uss. finden, welche von den drei englischen Handschriften zweifellos die jüngste ist" ${ }^{2}$ ). Dies soll zwar nach Cornills

1) Ich löse Cornills Siglen auf, ohne dies besonders zu bemerken.

2) Ueber das Alter der für die Londoner Polyglotte benutzten Handschriften giebt Cornill 138 einige Bemerkungen. Falsch ist die Angabe, dafs Cant. aus dem Jahre 1066 stamme. Cornill folgt hier der Notiz Thorndikes im Variantenverzeichnisse S. 1. Aber Thorndike verbessert sich -selbst S. 49 : Cant. stammt aus dem Jahre 1485 der Griechen = 1174 n. Chr. Ueber Uss. worde ich unten Genaueres beibringen. 
Meinung kein Beweis sein, aber doch ein Vorurteil gegen a erwecken. Aber sollte hier Cornill nicht ein Irrtum untergelaufen sein? Ohne dafs er es ausdrücklich sagt, liegt doch bei ihm der Gedanke zu Grunde, dafs die jüngste Handschrift auch den schlechtesten Text biete; sonst könnte ja das Uebereinstimmen von a mit der jüngsten Handschrift keinen Verdacht gegen a erregen. Diese Voraussetzung ist aber falsch; Alter der Handschrift und Güte des Textes stehn bekanntlich nicht, wenigstens nicht immer, in genauer Correlation. Und wenn von mehreren Handschriften die älteste und die jüngste gegen die übrigen zusammenstimmen, so kann man daraus nie ein Vorurteil gegen die älteste, sondern nur für die jüngste ziehen. Denn aus dieser Uebereinstimmung folgt ja, dafs ein solcher Text, wie ihn die jüngste Handschrift bietet, schon lange Zeit, in unserem Falle schon 1000 Jahre, früher existiert hat, als die Handschrift geschrieben wurde, dals also in diesen 1000 Jahren der Text sehr treu überliefert ist.

Von den 195 Varianten rechnet Cornill ,die sieben, nicht den Text selbst, sondern Ueber- und Beischriften betreffenden Abweichungen ab und ferner, um das Verhältnifs für a nicht zu ungünstig zu gestalten, diejenigen 14 Stellen, an welchen wenigstens noch eine der drei englischen Handschriften zufällige Lücken gs ergänzt." Die Abrechnung jener 7 Stellen ist durchaus in der. Ordnung, aber gegen die Abrechnung dieser 14 Stellen mufs ich Einspruch erheben. Cornill will untersuchen, ob a oder $g$ einen besseren Text habe, und dann rechnet er 14 Stellen, an welchen a Worte hat, die in $\mathrm{g}$,zufällig fehlen", an welchen also a das Richtige, $\mathrm{g}$ das Falsche hat, nicht mit, und zwar „um das Verhältnifs für a nicht zu ungünstig zu gestalten". Das vermag ich nicht zu verstehn.

Es bleiben 174 Varianten. „Unter diesen sind die 40 mit einem Kreuze bezeichneten nur innersyrische Verschiedenheiten ... Es bleiben demnach 134 Varianten, 
mit welchen wir hier zu rechnen haben. Von diesen 134 Varianten sind aber 86, also fast genau zwei Drittel, derart, dafs sie gegen die übrigen Recensionen [?] der Peschito mit dem massorethischen Text gehn." Hieraus folgert dann Cornill, „dals a nàch dem massorethischen Texte, wenn auch nicht gerade systematisch überarbeitet, so doch in ausgedehnter Weise corrigiert und geändert ist." Wenn wir dies Resultat Cornills auf seine Richtigkeit prüfen wollen, wird es.. also darauf ankommen, jene 86 mit $\mathfrak{M}$ übereinstimmenden Stellen zu untersuchen. Cornill hat sie durch Sternchen kenntlich gemacht; ich habe sie nachgezählt, aber nur 84 gefunden, als 85. ist offenbar Ezech 25, 17 zu zählen, über die 86. bin ich unklar.

Unter diesen 85' Varianten beruht Eine auf einem

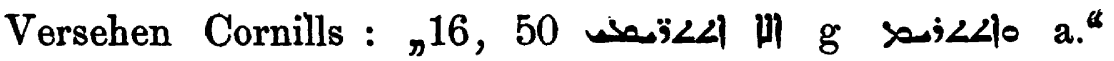
Wenn a so; 2410 hätte, so würde dies allerdings genauer mit dem hebräischen ותגבהינה übereinstimmen, als gs

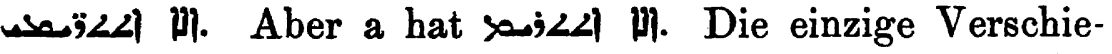
denheit ist die rein orthographische, dals $\sim$ und die Pluralpunkte in a fehlen.

Hinsichtlich der übrigen 84 Varianten ist Cornill zunächst der Vorwurf zu machen, dals er dieselben blofs gezählt, nicht gewogen hat. Er sayt blofs : In diesen Fällen stimmt a gegen $g$ mit $\mathfrak{M}$ überein. Aber er hat vergessen, die Frage zu stellen : Woher kommt es, dals a gegen $g$ mit $\mathfrak{M}$ übereinstimmt? Denn es ist doch nicht blofs möglich, dafs in diesen Fällen die Peschita ursprünglich von $\mathfrak{M}$ abwich, und a erst nachträglich nach $\mathfrak{M}$ corrigiert wurde, sondern es giebt auch die andere Möglichkeit, dafs a den ursprünglichen, mit $\mathfrak{M}$ übereinstimmenden Text der Peschita bietet, während die Verschiedenheit gs von $\mathfrak{M}$ erst durch spätere Verderbnis des Textes hervorgerufen ist. 
Cornill bemerkt

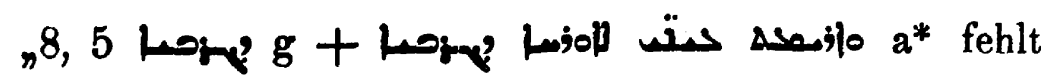
auch bei Cant. Poc. Uss. Lee."

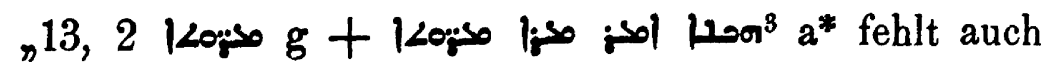
bei Cant. Poc. Uss. Lee."

„16,57 مصته a a* fehlt bei allen übrigen."

In diesen Fällen hat, wie jeder sieht, und wie auch Cornill selbst S. 137 sagt, ein Homoioteleuton den Ausfall der betreffenden Worte in $g$ verursacht. Also kann man hieraus nur folgern, dafs $g$ verderbt ist, nicht, dafs a einen nach $\mathfrak{M}$ corrigierten Text bietet. Wenn Cornill übrigens als Zeugen für das Fehlen der betreffenden Worte auch Cant. Poc. Uss. Lee anführt, so beweist Lee als Abdruck von $g$ nichts. Cant. Poc. Uss. führt er an, weil im Londoner Variantenverzeichnisse keine Abweichungen dieser Handschriften von $g$ notiert sind, und weil er annimmt, dafs kein Grund vorliege; an der Genauigkeit der Collation Thorndikes zu zweifeln (S. 138). Aber schon 1821 hat S. Lee, wie oben erwähnt, die Ungenauigkeit derselben für Gen 1-8 durch eine Liste der in diesen Kapiteln übergangenen Varianten nachgewiesen; er schliefst mit den Worten : „I shall only add that readings of much greater extent and importance than the above, have been omitted by Thornedyke in his collations made for the London Polyglott." Für die Psalmen ist dies durch meine obige Untersuchung bestätigt. Also sind argumenta e silentio hier unguiltig, und Cant. Poc. Uss. können trotz Thorndikes Schweigen recht wohl die bei $g$ fehlenden Worte haben.

Nach Cornill 139 f. ist 39, 18 il| g Uss. nur innersyrische Verschreibung für dem versieht Cornill مونتما in dem Variantenverzeichnisse mit einem Sternchen, statt mit einem Kreuze. 


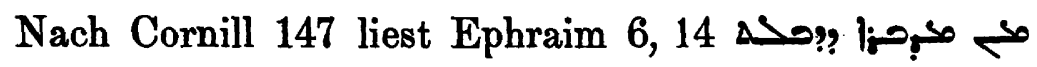
mit a Cant. Poc. Uss. gegen g Lee. Hier stehn fünf Zeugen gegen Einen, und doch lärst Cornill sich nicht irre machen, sondern rechnet $6,14 \mathrm{zu}$ den Stellen, welche beweisen sollen, dafs a nach $\mathfrak{M}$ corrigiert ist.

Wie hier, so stimmen auch an den Stellen 18, 17 und 27, 14 Cant. Poc. Uss. mit a gegen $g$ überein. Hierdurch ist eine Correctur bei a ausgeschlossen; man müfste sonst auch annehmen; dafs Cant. Poc. Uss. ebenso corrigiert seien. Also beweisen auch diese Stellen nicht für eine Correctur as, wohl aber gegen die Richtigkeit gs.

Liefs sich in diesen 7 Fällen leicht entscheiden, was ursprünglich, was verderbt sei, so ist es schwieriger in den übrigen 77 Fällen, wenigstens mit den von Cornill herangezogenen Hülfsmitteln. Cornill hat aber Ein Hülfsmittel gar nicht verwertet : die Bibel von Urumia, deren Bedeutung ich oben (unter I) auseinandergesetzt habe. Diese stimmt nicht nur an den angeführten 7 Stellen, sondern auch an weiteren 32 völlig mit a überein : 1, 18 [hier entspricht übrigens dem sondern Fog مo]. 7, 19. 14, 1. 16, 12. 47. 56. 17, 6 poor. 20, 5. 6 bis. 15. 23, 7. 21. 24, 10. 26, 3. 4. 27, 6. 26. 33. 31, 16. 33, 20. 37, 24. 38, 14. 16. 42, 15. 43, 10. 18. 44,25 . 45, 13. 46, 1. 48, 17. 24 . $\mathrm{Da}$ in diesen Fällen zwei von einander sicher unabhängige Texte, der ostsyrische $u$ und der westsyrische a, die gleichen, mit $\mathfrak{M}$ übereinstimmenden Lesarten haben, so ist es von vorn herein so gut wie sicher, dafs dies die alten Lesarten der Peschita sind. Man mülste sonst schon annehmen, dafs ein Ostsyrer und ein Westsyrer unabhängig von einander auf den Gedanken gekommen seien, die Peschita nach $\mathfrak{M}$ zu corrigieren, und dafs sie unabhängig von einander diese 32 Stellen in der gleichen Weise corrigiert haben. Das wird aber wohl niemand zu glauben geneigt sein. 
Ich worde nan diese 32 Stellen nicht alle einzeln besprechen; dies würde zu weit fübren und von geringem Nutzen sein. Ich bemerke aber, dafs zu denselben gerade zwei von den drei Stellen gehören, welche Cornill selbst für die wichtigsten erklärt : 16, 12, wo u nur Mo;هo statt Nojoo a hat, und 48, 17. An der Stelle 38, 14 war es übrigens leicht, das Richtige auch ohne u zu finden. Hier fügt $\mathrm{g} \mathrm{zu} \underset{\mathrm{z}}{\mathrm{r}}$ ein hinzu; dies erklärt sich daraus, dafs Gog und Magog sehr häufig zusammengenannt werden, nicht nur in der Bibel, sondern auch in dem allbekannten Gedichte über den gläubigen König Alexander und das Thor, welches er gegen Gog und Magog machte.

Wenn wir diese 32 Stellen abziehen, so bleiben 45 übrig. An 41 dieser Stellen stimmt a mit g gegen a überein. Es ist also wahrscheinlich, dals $\mathrm{g} u$ hier das Richtige bieten, wenn auch nicht ganz sicher. Denn die amerikanischen Missionare können zwar a nicht benutzt haben, weshalb Uebereinstimmungen von a gegen $g$ beweisen; aber sie könnten vielleicht Lees Text für ihre Ausgabe mit benutzt haben. Es ist also die Heranziehung neuer Zeugen für diese Stellen sehr erwünscht. Vorläufig ist anzunehmen, dafs $\mathrm{g} u$ hier das Richtige haben, und es fragt sich nun: Werden wir durch diese Stellen gezwungen, eine Correctur as nach $\mathfrak{M}$ zu behaupten? Sehen wir die betreffenden Varianten durch, so finden wir darunter sehr viele, welche Cornill, wenn nicht gerade a etwas genauer als $\mathrm{g}$ mit $\mathfrak{M}$ übereinstimmte, gar nicht mit einem Sterne, sondern mit einem Kreuze versehen und dadurch als innersyrische Verschiedenheiten gekennzeichnet haben würde. Neunmal (17, 6 sasoo. 18, 9. 23, 10. 26, 1. 29, 11. 32, 21. 36, 38. 43, 25. 48, 8) besteht die Verschiedenheit in der Hinzufügung oder Weglassung der Copula $\bullet$, dreimal $(8,3$. 28, 19. 29, 6) in dem Wechsel zwischen 0 und , zweimal 
$(7,4.15)$ in dem Wechsel zwischen Singular und Plural, zweimal $(11,21.30,13)$ in der Umstellung eines Wortes.

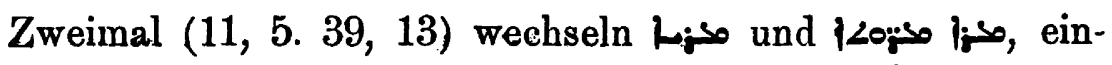

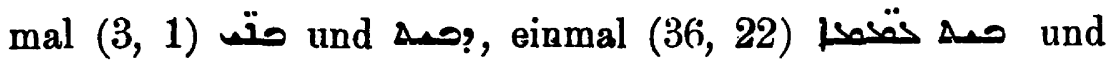

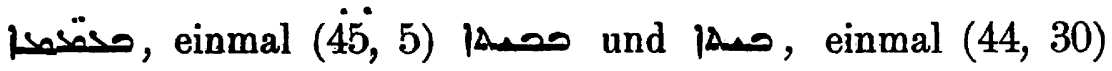

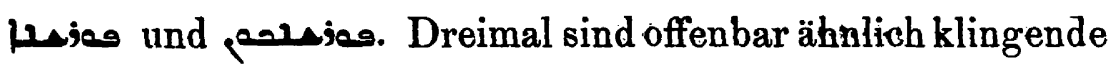

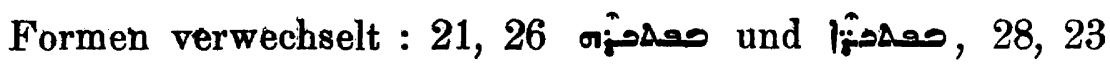

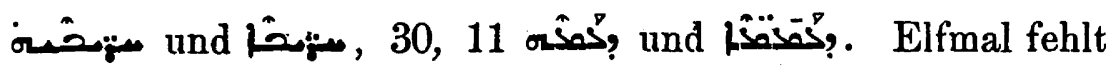
je ein Wort bei $a$ :

6, 8 das zweite

7, 9 (dies Wort kommt in den Versen 3-9 elfmal vor)

14, 17 das zweite in

| 16,

17, 3

17, 12 منر (Anrede an Ezechiel)

27, 25 das zweite 2

$32, \quad 3$ io

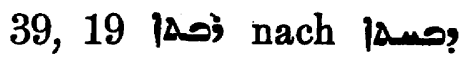

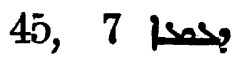

45, مب: 18 .

Umgekehrt hat a dreimal je ein Wort mehr als $\mathrm{gu}$ :

20, 12 (in demselben Verse kommen schon zwei إ) vor)

21, 15 1kommt in v. 14 schon einmal vor) $32,27 \mathrm{p}$.

Diese 39 Varianten sind solche, wie sie sich stets zwischen zwei Handschriften finden. Für die Annahme einer absichtlichen Correctur sind sie viel zu unbedeutend. Dazu kommt noch, dafs manchmal die Uebereinstimmung von a mit $\mathfrak{M}$ recht problematisch ist, z. B. 


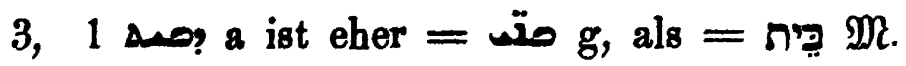

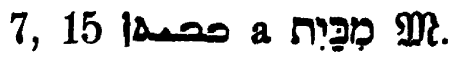

20, 12 כי אני יהוה = gu ist סمبی wie Cornill angiebt, hat aj weicht ab.

28, 23 wק a 2 a $\mathfrak{M}$.

29, 11 Hol g u entspricht dem hebräischen nauer als Holo a.

32, 3 | 3 | setzung der hier im Anfange einer Rede sehr auffälligen Copula sein, also stimmen gu gegenauer mit $\mathfrak{M}$, als a, in welchem in fehlt.

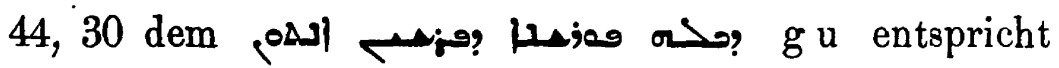
in $\mathfrak{M}$ וכל תרומח כל מכל חרומתיכם ; das Suffix in

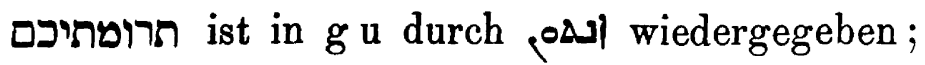
wenn a gast, so ist es zweimal übersetzt.

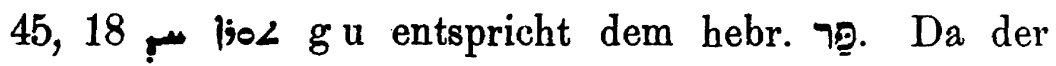
Artikel im Syrischen der Peschita schon seine Bedeutung verloren hat, so wird $7 \underline{\underline{0}}=$ ein Stier durch den stat. emph. j;०L übersetzt; da aber dies doch auch noch der Stier heifsen kann, so wird zur Verdeutlichung مب hinzugesetzt. g u $=\mathfrak{M}$, nicht a, in welchem مبر fehlt.

Die beiden einzigen gröfseren Varianten zwischen $g u$ und a sind

39, 4 بمطس g a lap? a

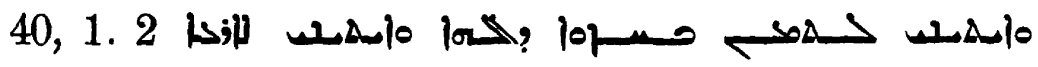

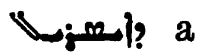

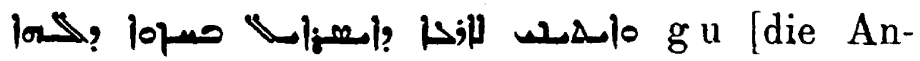
gabe Cornills ist hier ungenau].

In diesen beiden Fällen bin ich geneigt anzunehmen, dafs a trotz g u Recht hat. Denn 39, 4 konnte der gewöhnliche Ausdruck „Vögel des Himmels“ leicht an die 
Stelle von „geflügelte Vögel ${ }^{\star}$ treten; und $40,1.2$ sieht mir die Lesart von $\mathrm{g} u$ wie eine Zusammenziehung der Lesart as aus. Doch enthalte ich mich eines abschliefsenden Urteils. Jedenfalls berechtigen uns auch diese 41 Varianten nicht, eine Correctur as nach $\mathfrak{M}$ anzunehmen.

Nun bleiben noch 4 Stellen übrig : 12, 13. 25, 17. 36,7 . 38, 7. Um mit der letzten zu beginnen, so hat hier

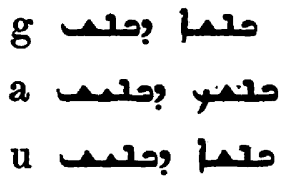

Cant. Poc. Uss. haben פوבسي.

Fest steht somit, dafs a gegen g richtig وصد / وحس ; hat g giebt auch gar keinen Sinn. Zweifelhaft könnte sein, ob معر richtig ist; vorläufig hat ersteres die grö fsere Wahrscheinlichkeit für sich.

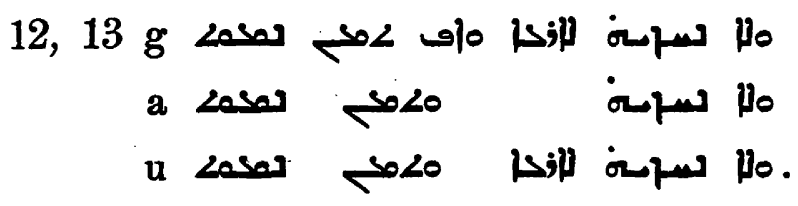

Ich glaube, dafs u die richtige Lesart bietet, und dafs der Schreiber von a isj, nachdem er es dicht vorher schon einmal geschrieben hatte, hier ausliefs.

36,7 lo g Hon $u$ > a.

Hier wird $g$ das Richtige haben, in $u$ Hor unter dem Einflusse des darauf folgenden $\mid \boldsymbol{H}$ aus lor verderbt sein, und in a lo durch ein Versehen (vgl. 32,3) ausgefallen sein.

25, 17 ist die Stelle, welche Cornill für die bezeichnendste hält. „Hier, am Ende von Cap. 25, also genau an der Stelle, an welcher die Massora bemerkt חצי הספר,

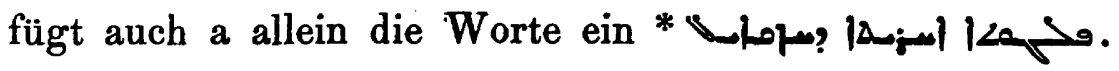

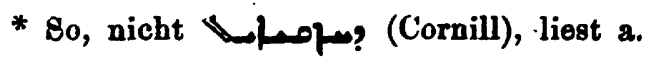


Dics Zusammentreffen ist um so auffallender, als bei 25,17 durchaus kein Sinnesabschnitt, kein naturlicher Theilungspunkt vorliegt, während das Ende von Cap. 24 fast unmittelbar vorher nicht nur nach der Capitelzahl, sondern auch sachlich, die Mitte von Ezechiels Buch bildet." Dafs hier wieder ein argumentum e silentio vorliegt, will ịch nicht zu stark betonen. Die Worte könnten indessen von den Herausgebern von $\mathrm{g} u$ als nicht zum Texte gehörig fortgelassen sein. Aber selbst wenn sie auch in den zu Grunde liegenden Handschriften fehlen, beweist dies, dafs a nach $\mathfrak{M}$ corrigiert ist? Die Peschita ist ja nach Cornills eigener Ansicht unter dem mitwirkenden Einflusse jüdischer Tradition entstanden. Also kann die Beischrift auch ursprünglich und erst später fortgelassen sein. Und sie findet sich sicher wenigstens noch in Einer anderen Handschrift. British Museum Add. 12135 foll. 1-43 (Wright, Catalogue 1, 24-26) aus dem Jahre 726 n. Chr. enthält Ezech 26 48. An der Spitze steht oو

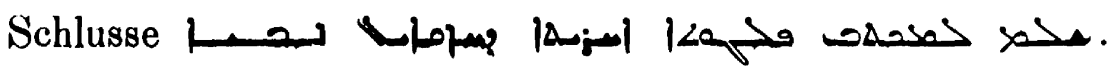
Hieraus sehen wir, dafs die Bemerkung in der Peschita der in $\mathfrak{M}$ zwar ähnlich, aber doch nicht gleich ist. In $\mathfrak{M}$ ist „Mitte des Buches“ eine blofse Randbemerkung, in der

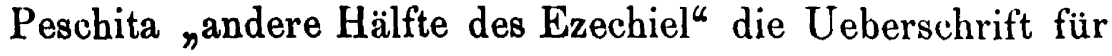
cap. 26-48. Dafs man gerade am Ende von cap. 25 den Einschnitt machte, kann man aus dem Einflusse jüdischer Tradition erklüren. Aber in der Peschita hat diese Einteilung nicht blofs, wie in $\mathfrak{M}$, einen theoretischen, sondern unter Umständen auch einen praktischen Zweck. Bei der Zerlegung des Buches des Propheten Ezechiel in zwei gleich grofse volumina begann man das zweite volumen mit $26,1$.

Somit habe ich die Varianten, welche beweisen sollen, dafs a in ausgedehnter Weise nach $\mathfrak{M}$ corrigiert und geändert ist, geprüft. Als Resultat ergiebt sich, daf's Cornills 
These nicht haltbar ist. An vielen Stellen bietet a gegen g das Richtige, an manchen hat a Fehler. Natürlich, denn unfehlbar ist keine Handschrift. Ich mufs noch einen letzten Grund Cornills besprechen, nämlich den, „dafs a auch in der Abtheilung von Cap. 20 gegen alle übrigen Zeugen mit der Massora geht, indem er Cap. 21 nach 20, 44 beginnen läfst, während g Lee und vermuthlich auch Cant. Poc. Uss. noch die fünf ersten Verse von Cap. 21 zu 20 ziehn, und wie LXX Cap. 21 erst mit 21, 6 hebr. beginnen lassen." Ich mufs gestehn, dafs mich dieser Satz Cornills in einige Verlegenheit gebracht hat; denn ich wufste anfangs nicht recht, worauf er sich beziehen soll. Die gewöhnliche Einteilung des Ezechiel bei den Syrern, welche sich auch bei dem Araber der Pariser. Polyglotte findet

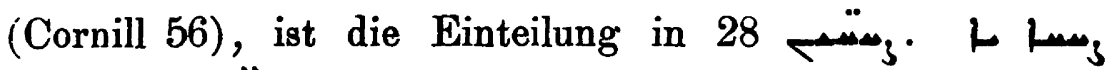
$=$ = الامحاح der Geheimnisse (Cod. gott. or. 18 ${ }^{\mathrm{a}}$ ), wie in dem Araber nach 20, 44 . $u$, überhaupt in dieser Beziehung unzuverlässig, lä[st L $\mathrm{L}_{3}$ fälschlich nach 20,49 beginnen. Aber a hat diese Einteilung in ${ }_{3}$ nicht, Cornill kann sie also nicht meinen. In a findet sich eine andere, von späterer Hand beigeschriebene Einteilung in $22 \mathrm{Kapitel;}$ von diesen beginnt $\bar{a}$ nach 20,44. Aber da sich in $g$ nichts mit dieser Einteilung Vergleichbares findet, und da Cornill auch nicht von dem Anfange von cap. 15, sondern von cap. 21 spricht, so wird er auch dies nicht meinen. Es bleibt also nichts übrig, als die Annahme, dafs Cornill geglaubt hat, die Einteilung in 48 Kapitel gehöre den Handschriften der Peschita an, und es sei ein in den Handschriften begründeter Unterschied, dafs a das 21. dieser 48 Kapitel mit $\mathfrak{M}$ nach 20,44, g mit LXX erst 5 Verse später beginnen läfst. Wenn dem aber so ist, so ist des Rätsels Lösung nicht schwer zu finden. Die 
Kapitel- und Verszahlen sind weder in a, noch in $g$ ur-. springlich. In a hat sie Ceriani, in $\mathrm{g}$ Gabriel Sionita eingesetzt. Da sich Ceriani nach der Ausgabe des E. van der Hooght richtete (Praefatio $8^{3}$ ), Gabriel der in der römischen Kirche üblichen Einteilung der Vulgata (hier $=\mathrm{LXX})^{1}$ ) folgte, so stimmt a mit unsern Handausgaben von $\mathfrak{M}$, $g$ mit $\mathrm{LXX}$ überein. $\mathrm{Zu}$ untersuchen, was Cant. Poc. Uss. haben, dürfte demnach überflüssig sein.

\section{IV.}

Hiermit ist die Untersuchung betreffs a beendigt. Das Resultat derselben wirft aber weiter ein Licht auf die Beurteilung von Uss. Hatte Cornill bemerkt, dafs sich gerade zwischen a und Uss. „eine auffallend grofse Zahl von Berührungen finden", und hat die bisherige Untersuchung ergeben, dafs a doch an manchen Stellen das Richtige hat, so ist es vielleicht möglich, dafs auch Uss. nicht ganz so schlecht ist, wie Cornill meint. Rechne ich die vier Stellen, an welchen Cornill unberechtigter Weise e silentio auf die Lesart von Uss. schliefst, nicht mit, so erscheint Uss. in Cornills Variantensammlung ${ }^{2}$ ) im Ganzen 49 mal. Unter diesen 49 Stellen sind aber 39, an welchen Uss. mit a u gegen $\mathrm{g}$ übereinstimmt : $1,25.6,11 \mathrm{f}$. $14.8,6 \mathrm{f}$. [hier

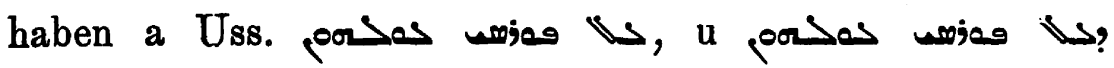
als Ueberschrift von v. $7 \mathrm{ff}$.] 9,8 . 11, 10. 12. 21. 13, 15 f. $14,1.4$. $16,32.51$ f. 17,6 poor. $18,17.20,5.6$. 15. 21,32 ه. 22, 28. 23, 7. 28. 44. 24, 12. 24-27.

1) Uebrigens zählt auch im hebräischen Texte der Pariser Polyglotte das 20. Kapitel 49 Verse, was Walton in der Londoner Polyglotte nicht geändert hat.

$\left.{ }^{2}\right)$ Die von Cornill nicht aufgeführten Varianten von Uss. lasse ich unberücksichtigt, da sie eine weitläufigere Behandlung erforden würden, und da, um zu einem abschliefsenden Urteil über Uss. zu kommen, Thorndikes Collation doch nicht ausreicht. 
27, 14. 29, 12. 30, 12. 32, 30 [bei Cornill Druckfehler $32,20]$. 32, 31 f. $33,18.19$. 20. 36, 1. 38, 7. 43, 3. 11. 44, 25. 45, 13. Hierbei sind diejenigen Fälle mitgerechnet, in denen sich in $\mathrm{g}$ Lücken finden, welche Uss. $=\mathrm{a} u$ richtig ergänzt, . sowie auch 2 Fälle $(1,25.32,31$ f.), in welchen Cornill aus infolge von $\delta \mu o \iota \tau_{\varepsilon} \lambda \varepsilon v \tau o \nu$ entstandenen Fehlern sehr gut schliefst, dafs a und Uss., resp. deren Vorlagen, ursprünglich übereingestimmt haben. Unter den 10 übrig bleibenden Stellen sind 3, wo Uss. allein steht : 16, 47. 27, 26. 33. Diese 3 Lesarten von Uss. beruhen sicher auf Schreib- oder Druckfehlern. Auch 27, 20. 39, 18 liegen kleine Fehler in Uss. vor. Unbedeutend ist die Variante 21, 32 أو $\mathrm{g}$ ف a Uss. Dreimal $(12,6.42,3$. 48, 6) stimmt Uss. mit a Cant. Poc. gegen g u, einmal $(17,6)$ mit a Cant. Poc. Ephraim gegen g u überein; hier ist ein Heranziehen weiterer Zeugen nötig. Im Ganzen sehen wir, dafs Uss. in der überwiegenden Mehrzahl der Fälle mit a u gegen $g$ übereinstimmt.

Diese Thatsache gewinnt aber für uns eine noch gröfsere Bedeutung, wenn wir uns an den Ursprung von Uss. ${ }^{1}$ ) erinnern. Die noch kürzlich von Edv. Stenij, De Syriaca libri Iobi interpretatione. I. Helsingfors 1887 [mir bekannt geworden durch Gottheils Aufsatz S. 28] S. 27 citierte Angabe Waltons in den Prolegomena 892, Uss. sei "ex codice Patriarchae Alexandrini" abgeschrieben, ist falsch. Aus dem bei Parr, Life and letters of Archbishop Usher (London 1686) ${ }^{2}$ ) unter No. 69 abgedruckten Briefe

1) Mit Uss. meine ich hier immer die Ussher'sche Handschrift des alten Testamentes ohne die Psalmen, welche in Thorndikes Variantenverzeichnisse im Pentateuche Uss. 2, sonst Uss. genannt wird. Sie ist wohl zu unterscheiden von dem Pentateuche und dem Psalter Usshers, welche bei Thorndike auch Uss. heifsen.

2) Ich weifs zwar, dafs der Briefwechsel Usshers in dem 15. und 16. Bande der seit 1847 in Dublin erschienenen Gesamtausgabe der

Zeitschrift f. d. alttest. Wiss. Jahrgang 9. 1889. 
des in Aleppo angesiedelten, englischen Kaufmannes Thomas Davies an Ussher vom 29. August 1624 englischen Styls $=8$. September 1624 gregorianischen Styls ${ }^{1}$ ) ergiebt sich, dafs Ussher den Davies beauftragt hat, ihm das syrische alte Testament „according to the Hebrew" ${ }^{2}$ ) zu beschaffen.

Werke Usshers von Elrington vermehrt herausgegeben ist, bitte aber zu entschuldigen, dafs ich diese Ausgabe nicht benutzt habe, da sie weder in der Göttinger, noch in der Berliner Königl. Bibliothek vorhanden ist.

1) Die Engländer hatten damals noch den julianischen Kalender und begannen aufserdem das Jahr mit Mariä Verkündigung (25. März), sodals z. B. das englische Jahr $1624=4$. April 1624 bis 3. April 1625 gregorianischen Styls ist. Dafs Davies nach dem englischen Style datierte, beweist Gwynn, On a Syriac MS. in den Transactions of the Royal Irish Academy [Lagarde, Mittheilungen 2, 187] 27, 281.

$\left.{ }^{2}\right)$ Durch diesen Zusatz unterscheidet Ussher die Peschita von dem aus dem Griechischen übersetzten hexaplarischen Syrer, von welchem man seit Andreas Masius wufste. Auch den hexaplarischen Syrer sollte Davies für Ussher aufsuchen, aber er hatte hierin kein Glück. Er schreibt letter $81:{ }_{n} \mathrm{I}$ have sought the Old Testament in that Tongue, which is out of the Greek, and distinguished by certain Marks and Stars : but I cannot hear of any such ${ }^{4}$. Später $(12 .=22$. Nov. 1639) gab Ussher dem Christianus Ravius denselben Auftrag. Letter 305 : ${ }_{n}$ Libri quos pro me requiri velim, hi sunt: Vetus Testamentum Syriacum, non ex Hebraeo factum (illud enim jam habeo) sed ex Graeco versum, atque obelis et asteriscis distinctum ...". Christian Raue, derselbe, welcher durch seine aus der Complutensis und Regia zusammengeschriebene, 1 Joh 5, 7 enthaltende Pergamenthandschrift des neuen Testamentes eine traurige Berühmtheit erlangt hat, machte in den Jahren 1639-1642 von England aus eine Orientreise (nach Smyrna, Konstantinopel, durch Kleinasien), zu welcher ihm Ussher eine Unterstützung von jährlich $24 £$ aussetzte unter der Bedingung, dafs Raue für ihn Handschriften des hexaplarischen Syrers, der Briefe des Polykarp und Ignatius in syrischer Uebersetzung $u$. a. aufsuche und ihm von Zeit zu Zeit Bericht über seine Reise abstatte (letter 305, womit zu vergleichen, was Raue selbst in seinem "Spolium Orientis" auf der zweiten Seite des Bogens E sagt : ${ }_{n}$ Ad has tot ac tantas impensas vel solus Jacobus Usserius quinque ArchiEpiscoporum in Hiberniâ Primas Armachanus, Vir supra laudem et titulos, Mille Imperiales gratuito mihi misit in subsidium ${ }^{4}$ ). Jedoch scheint Ussher mit Raue wenig zufrieden zu sein. Er schreibt am 30. Sept. = 10. Oct. 1640 an Samuel 
Davies konnte nur Ein Exemplar des ganzen alten Testamentes auffinden, welches jedoch nicht verkäuflich war. Letter 83 : „Amongst all the Caldeans that lay in Mount Libanus, Tripoly, Sidon, and Jerusalem, there is but only one old Copy of the Old Testament in their Language extant, and that in the custody of the Patriarch of the Sect of the Maronites, who hath his residence in Mount Libanus, which he may not part with upon any terms". Von diesem in dem Kloster Kenobin (oder Qannobîn Lagarde, Mittheilungen 2, 187) $=$ Kovvó $\beta \iota o v$, dem Sitze des Patriarchen, damals Johanns XI., aufbewahrten Exemplare liefs Davies durch „Joseph, Sohn des David, aus der Stadt Van im Libanon", welcher von einem jakobitischen Priester und Mönche Cyriacus unterstützt wurde, eine Abschrift nehmen. (Das Nähere siehe bei Gwynn, Transactions of the Royal Irish Academy 27, $283 \mathrm{f}$.) Die Vollendung dieser Abschrift, welche Davies dem Ussher am 14. März $1627=24$. März 1628 mitteilt, nahm 17 Monate in Anspruch (letter 120); die Handschrift stammt also aus den Jahren 1626-1628. Sie umfafst das ganze alte Testament "wanting only the Book of the Psalms, and the second of Esdras" (letter 120). Uss. ist also eine Abschrift aus dem Exemplare des Patriarchen der Maroniten. Dieser aber führt den officiellen, ihm vom Papste verliehenen Titel „Patriarch von Antiochien“, und mit diesem Titel wird Johann XI. auch in Uss. ausdrücklich bezeichnet (Gwynn 283). Also ist statt „Patriarchae Alexandrini“ in Waltons Prolegomena „Patriarchae Antiocheni“ zu lesen. Das Richtige giebt übrigens Walton selbst an in dem 1652 ausgegebenen Prospecte der Londoner Polyglotte (abge-

Hartlib, letter 306 [bei Parr S. 624 ist statt CCCVI fulschlich LX gedruckt] : "I gave order unto Mr. Ravius, to procure me a Copy of Ignatius his Epistles in the Syriack Language, to which he hath returned me no full answer." Jedenfalls hat Raue die ersehnten Handschriften nicht gefunden. 
druckt bei Todd, Memoirs of the Life of Walton I $31 \mathrm{ff}$.), nach welchem die Polyglotte die Peschita enthalten soll "corrected by Copies transcribed out of the most ancient and perfect MSS. remaining with the Patriarch of Antioch" (Todd I 38). Irrefuhrend ist hier nur der Plural, welcher den Schein erweckt, als seien alle für die Londoner Polyglotte verglichenen Handschriften aus Exemplaren des Patriarchen von Antiochien abgeschrieben. Dagegen palst die Beschreibung gerade auf Uss. vollständig. Ich bemerke noch, dafs auch Hottinger in seinem 1658 erschienenen Promtuarium S. 282, indem er die Worte Waltons in den Prolegomena im Ganzen reproduciert, doch richtig "ex Patriarchae Antiocheni exemplari" sagt, und dafs sich die genaueste Beschreibung der Handschrift aus alter Zeit in den von Hottinger citierten, 1644 in London erschienenen nAnimadversiones sacrae ad textum Hebraicum Veteris Testamenti“ des A. Bootius findet. Dieser, welcher Uss. um 1640 in Dublin benutzte (vgl. die Widmung und Vorrede des Buches), berichtet im "Index autorum" zum Worte nSyrus", dals Ussher "multo auro totum Vetus Testamentum (exceptis Psalmis, quia eos jam ante typis vulgatos habebamus) e libro Patriarchae Antiocheni in monte Libano describi sibi curaverat anno 1527 [lies 1627] per quendam Josephum filium Davidis". Am 18. = 28. Juli 1653 bat Brian Walton den Ussher, ihm diese Handschrift zu schicken, damit sie Thorndike für die Londoner Polyglotte collationieren könne (Parr, letter 276), und Ussher erfüllte seinen Wunsch. $\mathrm{Da}$ indessen Ussher schon 1656 starb, behielt Walton sie in seiner Bibliothek, und aus dieser ist sie nach Waltons Tode, ebenso wie 5 andere von Walton dem Ussher entliehene Handschriften (drei samaritanische Pentateuche, ein syrischer Pentateuch, ein syrischer Psalter), in die Bodleiana zu Oxford übergegangen, wo sie seitdem aufbewahrt wird (Catalogus librorum ms. Angliae et Hiberniae, Oxon. 1697, I 1, $156^{2}$, vgl. auch Gwynn 285 f.). 
Uri und Payne Smith führen sie in ihren Katalogen der syrischen Handschriften an erster Stelle auf.

Wenn wir Davies' Angabe trauen dürfen, so war die Handschrift, aus welcher Uss. abgeschrieben wurde, eine alte. Der Patriarch bewahrte sie in seinem eigenen Kloster auf und wollte sie um keinen Preis verkaufen; dies beweist, dafs sie von den Maroniten hochgeschätzt wurde. Damit gewinnt auch die Usshersche Handschrift, welche überdies eine recht genaue Abschrift zu sein scheint, an Wert. Am wichtigsten aber ist es für uns, dafs Uss. die Abschrift einer maronitischen Handschrift ist. Ich habe oben aus der Kirchengeschichte geschlossen, dafs die Gesamtheit der syrischen Bibelhandschriften in eine ostsyrische und eine westsyrische Gruppe zerlegt werden mufs. Jetzt schliefse ich weiter, dafs, da die Westsyrer sich später in Jakobiten, Melchiten und Maroniten getrennt haben, die westsyrische Handschriftengruppe weiter in drei Familien, die jakobitische, melchitische und maronitische, einzuteilen ist. Wiederum ist es hier unwahrscheinlich, dafs diese Familien sich gegenseitig beeinflufst haben sollten. Denn die Jakobiten und Melchiten standen sich feindlich gegenüber, und die Maroniten wichen von jenen nicht nur dogmatisch ab, sondern waren auch in ihren Felswohnungen im Libanon zu sehr abgeschlossen, um von aufsen etwas Neues anzunehmen. Demnach gelten für die Beurteilung der Varianten zwischen den verschiedenen westsyrischen Handschriften analoge Regeln, wie ich sie oben für die Beurteilung der Varianten zwischen den ostsyrischen und westsyrischen Texten aufgestellt habe. Wenn z. B. in den oben angeführten Stellen aus dem Psalter die melchitischen $\alpha \beta$ mit dem jakobitischen a gegen die jakobitischen $\gamma \delta$ u. s. w. übereinstimmen, so ist dadurch bewiesen, dafs die Lesarten von $\alpha \beta$ a die altwestsyrischen sind. Und wenn im Ezechiel der maronitische Text Uss. mit dem jakobitischen a gegen den jedenfalls auch westsyrischen $\mathbf{g}$ (von 
welchem ich nicht weils, ob er maronitisch oder jakobitisch ist) übereinstimmen, so folgt daraus, dafs Uss. a die altwestsyrischen Lesarten bieten. Wenn dann aber der so festgestellte altwestsyrische Text sich mit dem ostsyrischen u deckt, so beweist dies, dafs jene Lesarten nicht blofs altwestsyrisch sind, sondern der Zeit angehören, in welcher die syrische Kirche noch eine gemeinsame Geschichte durchlebte.

Hiernach läfst sich schon im voraus feststellen, welchen Weg der Herausgeber einer kritischen Peschitaausgabe einzuschlagen hat. Er mufs zunächst aus den jakobitischen, melchitischen und maronitischen Handschriften den altwestsyrischen Text, aus den nestorianischen Handschriften den altostsyrischen Text herstellen. Er mufs dann die Texte dieser beiden Gruppen vergleichen und daraus rückwärts auf den gemeinsyrischen Text schliefsen. Er darf endlich nicht aufser Acht lassen, dafs wir in der Handschrift des British Museum add. 14425 und in den Citaten bei Aphraates, Ephraim u. a. Zeugnisse aus der Zeit der ungetrennten syrischen Kirche haben. Auf diesem Wege wird sich mit grofser Sicherheit ein Text der Peschita gewinnen lassen, wie er im 5. Jahrhundert in Syrien umlief. Ein älterer Text wird im Ganzen nicht hergestellt werden können. Dals die Unterschiede zwischen den verschiedenen Handschriftengruppen sehr grofs sind, glaube ich allerdings nach den bisherigen Untersuchungen nicht; die Hauptveränderungen werden auch hier, wie überall, in den dunklen Zeiten der ersten Jahrhunderte liegen. Jedenfalls wäre es aber sehr wichtig, wenn auf dem angegebenen Wege ein möglichst alter Text hergestellt würde. Erst dann wäre die Peschita für die biblische Textkritik recht verwendbar. Denn die Ausgaben, welche wir bis jetzt haben, besonders a und $\mathrm{u}$, sind zwar dankbar hinzunehmende Vorarbeiten, aber auch nur Vorarbeiten. Die Hauptarbeit mufs jeder, der die Peschita jetzt benutzen will, selbst 
machen : er mufs durch Vergleichung von pl( $\left.\mathrm{l}^{\prime}\right)$ a u erst einen einigermalsen, aber auch nur einigermalsen, sicheren Text herstellen, wenn er nicht fortwährend im Ungewissen tappen will. Dafs aber jene vier (resp. fünf) Quartanten und Folianten sich bequem handhaben lassen, und dafs ihre Vergleichung eine leichte und angenehme Arbeit sei, wird niemand, der die Sache einmal gemacht hat, behaupten wollen.

Irre ich nicht, so wird bei der Feststellung des Peschitatextes Uss., obwohl sehr jung, als Abschrift einer älteren maronitischen Handschrift eine Rolle spielen. Besser freilich wäre es, wenn die Handschrift des Klosters Qannobîn, aus welcher Uss. copiert wurde, noch jetzt aufgefunden und nach Europa gebracht werden könnte, wie es Gwynn a. a. O. 284 für möglich hält. Dadurch würde natürlich Uss. überflüssig gemacht. So lange wir aber jene Handschrift nicht haben, müssen wir uns an Usshers Abschrift halten. Ich habe über dieselbe noch einige Mitteilungen zu machen.

Herbert Thorndike bemerkt in der dem syrischen Variantenverzeichnisse des 6. Bandes der Londoner Polyglotte vorangeschickten „Ad lectorem admonitio“, dafs an dem Rande von Uss. „subinde aspersae erant, manu nescio cujus, variantes e Gregorio quodam Syro, Scholiaste, ut opinor, quarundam Scripturae partium, lectiones, quas, quoties operae pretium videbatur, retulimus." Dieser Gregorius ist kein anderer als Gregorius Abulfarag Bar Ebhraya, und die Randnoten stammen aus dem |ㅟㅟ ; 이, wie jeder sieht, der sie mit den jetzt veröffentlichten Stücken dieses Werkes vergleicht. Wer der Urheber dieser Randnoten sei, darüber kann Thorndike keine Auskunft geben. Als er die Handschrift erhielt, standen sie schon darin. Sie müssen also vor dem Jahre 1653 geschrieben sein, in welchem Walton die Handschrift von Ussher erbat. Andrerseits können sie auch nicht von den syrischen Schreibern 
herrïhren, denn es findet sich z. B. zu Eccl 10, $1^{1}$ ) eine Verweisung auf Gregorius mit den lateinischen Worten „Vide Greg."; sie müssen also nach 1628 im Abendlande beigeschrieben sein. Ich glaube, dafs sie von Ussher selbst herstammen. Die Gründe für diese Annahme werde ich im Folgenden darlegen.

Ussher besafs ein Exemplar des lij ; $=10$. Dec. 1627 schreibt er an John Selden (letter 122): "I have had also another Book lately sent unto me from the East, intituled, Otzar Raza (or rather Razaja) a Treasury of Secrets, containing a brief Commentary in the Syriack Language, upon the whole Old Testament (excepting the Book of the Lamentations, Chronicles, Ezra, Nehemiah, and Esther) and likewise the New, those parts only excepted which are wanting in our printed Syriack Testaments." Er sagt hier, er habe dies Werk kürzlich aus dem Oriente, also durch Thomas Davies bekommen. In Davies' Briefen findet sich keine bestimmte Angabe über die Zusendung desselben. Es ist aber möglich, dafs Davies dies Werk in dem Briefe 81 (vom 16. Januar 1625 =26. Januar 1626) meint, wo er schreibt : „With the said Books [näml. den in der Peschita fehlenden Stücken des neuen Testamentes, dem syrischen Pentateuch und einer kleinen Abhandlung Ephraems] I have sent another

1) Thorndike : „Cap. X . . post versum secundum, ad oram Uss.

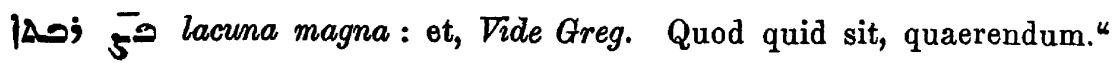
Das von Thorndike irrtümlich als $\left.\right|_{5}$ aufgefafste 5 ist natürlich $=10$, und die Randnote bedeutet : ${ }_{n}$ In einer Handschrift steht

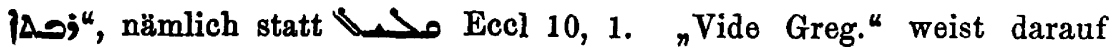
hin, dafs auch Gregorius jaj liest. Diese sich auch in u findende Variante, welche für Gottheil „komisch" und unerklärbar ist, rührt von jemandem her, welcher nicht verstand, wie ein wenig Thorheit schwerer wiegen könne, als grofse Weisheit, und daher "wenig" in nviel" andern zu müssen glaubte. 
in the same Tongue, which I humbly present your Grace." Wenigstens pafst es zu dieser Annahme recht gut, dafs Ussher in dem Briefe an Selden (vom December 1627) sagt, er habe es "lately" erhalten. Wenn die Annahme richtig ist, so hat Ussher die Handschrift etwa im Juni 1626 bekommen; $\ddot{d e n n}$ am 23. Juni $=3$. Juli 1626 schreibt er an Samuel Ward, er habe den syrischen Pentateuch, die fehlenden Stücke des neuen Testamentes und eine kleine Abhandlung von Ephraem "now" bekommen (letter 99). Dafs er hier den $|j| ;, 0 \mid$ nicht auch nennt, beweist nichts gegen jene Annahme. Denn dafs Ussher damals noch eine andere Handschrift bekommen hat, ist nach Davies' Briefe sicher. Ussher kannte den Inhalt derselben damals wohl noch ebenso wenig, wie Thomas Davies. Wie dem aber auch sein mag, jedenfalls war der $\mid$ i i ; 30 im December 1627 seit einiger Zeit in Usshers Händen. Und wie er damals über denselben an Selden berichtete, so später, 9. = 19. Juni 1632 und 13. = 23. Juni 1633, an Lud. de Dieu (letter 174. 175): Sowohl Selden, als de Dieu teilt er besonders über die chronologischen Tabellen des „Thesaurus secretorum "Genaueres mit; diese interessierten ihn, den Verfasser der Annales Veteris et Novi Testamenti, zunächst am meisten. Durch de Dieu hat dann wohl dessen Leydener College Constantin l'Empereur Nachricht über das Werk erhalten. Am 16. Nov. 1633 ${ }^{1}$ ) schreibt l'Empereur an Ussher : ${ }_{n}$ Cum in D[ignitatis] tuâ [lies : tuae] instructissimâ Bibliothecâ Syriacum in Biblia Commentarium esse intellexerim sive Thesaurum secretarium [lies : secretorum]; atque ab iis qui vetus Testamentum transferunt in linguam Belgicam rogatus sim, ut obser-

1) $\mathrm{Da}$ in der Provinz Holland seit 1582 der gregorianische Kalender eingeführt ist (L'art de vérifier les dates, depuis la naissance 1, 82), so sind die Daten der aus Leyden geschriebenen Briefe nicht umzurechnen. 
vationes ad loca difficiliora porro suppeditem : si optare liceret, istius Syriaci in Hagiographa et Prophetas posteriores Commentarii usum ad tempus concedi exoptarem" (letter 177). Ussher erfüllte seinen Wunsch, wie aus dem folgenden Briefe des l'Empereur (180) vom 29. Juni 1634 hervorgeht. Zugleich aber zeigt dieser Brief, dafs l'Empereur sich ziemlich enttäuscht fühlte. Aus dem ,Thesaurus secretorum" war freilich für die damals auf Beschlufs der Dordrechter Synode ausgearbeitete, neue niederländische Bibelübersetzung, die s. g. Staatenbibel, kein grofser Nutzen zu ziehen. „In veteri Testamento spei meae non respondet: licet subinde notatur [lies : notatu] digna animadvertam ... Nihilominus pro usu istius libri gratias habeo maximas : inprimis cum praeclara annotentur in Testamentum Novum." In dem dann folgenden Briefe des l'Empereur (182, vom 21. Dec. 1634) wird zum ersten Male der Name des Verfassers "Gregorius Syrus" genannt. Von 1634 bis Ende 1636 war die Handschrift in Leyden. In dieser Zeit nutzte

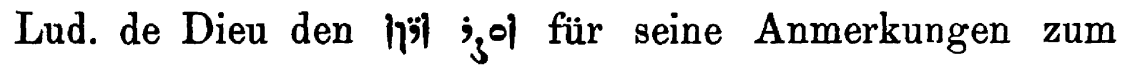
Römerbriefe aus. Denn dafs der in denselben (zuerst veröffentlicht Leyden 1646) oft citierte "Syrus Scholiastes" identisch mit Gregorius Bar Ebhraya ist, wie Hottinger, Promtuarium 285, da er selbst den 17i ; 01 nicht kannte, blofs vermuten konnte, bedarf jetzt keines Beweises mehr, da jeder die betreffenden Citate mit den kürzlich von Max Löhr herausgegebenen „Gregorii Abulfaragii Bar Ebhraya in epistulas Paulinas adnotationes" (Göttingen 1889) vergleichen kann. Am 15. = 25. Sept. 1636 schreibt Ussher an Constantin l'Empereur, er habe schon früher erfahren, dafs die neue Bibelübersetzung längst vollendet, und höre nunmehr, dafs sie auch gedruckt sei ${ }^{1}$ ); daher möge

1) Ausgegeben wurde sie zwar erst 1637 - die Vorrede ist vom 29. Juli 1637 datiert - , aber nach der Bemerkung des Druckers Paulus Aertsz van Ravensteyn am Ende des alten Testamentes ist wenigstens 
l'Empereur ihm „librum ... Syriacum Thesauri Secretorum inani titulo venditatum", wenn er denselben nicht weiter brauche, mit der ersten Gelegenheit zurückschicken (letter 192). Diesen Wunsch erfüllt Constantin l'Empereur alsbald; mit dem Briefe 197, welcher 8 Kal. Jan. An. 1637 $=$ Weihnacht $1 \dot{6} \dot{3} 6^{1}$ ) datiert ist, schickt er den Thesaurus secretorum durch den englischen Gesandten im Haag [William] Boswell ${ }^{2}$ ) an Ussher zurück. Später hat A. Bootius den 1;i ; 301 , ebenso wie Usshers altes Testament (vgl. oben), um 1640 in Dublin benutzt. Im „Index autorum" seines oben erwähnten Werkes giebt er darüber zum Worte "Gregorius Syrus" einen ausführlicheren Bericht. Er bedauert zunächst, dafs die Scholien des Gregorius oft sehr kurz sind und sich sehr viel mit grammatischen Kleinigkeiten abgeben. ${ }_{n}$ Habet tamen sparsim (praesertim in libro Psalmorum, ubi multo est quam in aliis libris plenior et prolixior) multa bona et utilia; quae ab aliis minoris momenti, ac heterogeneis, secreta, transcripsit ex eo omnia (postquam libro uti ipse desieram) amicus meus summus, D. Dudleius Loftus, filius Adami Loftus, Equitis

dieses schon 1636 gedruckt. Am Schlusse des neuen Testamentes und der Apokryphen finden sich solche Angaben nicht.

1) Dafs das Datum so zu verstehn ist, folgt daraus, dafs

1) l'Empereur den Brief beginnt : „Quas ad me 15 Septembris dedisti literas"; er sagt $n^{\mathrm{am}}$ 15. September", nicht ${ }_{n}$ am 15 . Sept. vorigen Jahres", also fallen beide Briefe in dasselbe Jahr,

2) dafs l'Empereur schreibt: „Vicissim vobis Catalogum librorum, quos D. Golius ex Oriente attulit, quemque D. Botius petierat, transmitterem : verum $D$. de Dieu, ut sibi id officii relinquerem voluit; ut qui ea de re literas a D[ignitate] tua accepisset." Diesen Katalog hat Ussher am 7. = 17. Juli 1637 schon seit längerer Zeit in Händen. "Literas tuas cum adjuncto librorum Academiae Vestrae Catalogo diu est quod accepi" (letter 195 an de Dieu).

2) Vgl. über ihn Leslie Stephen, Dictionary of National Biography V 440. Briefe William Boswells finden sich in Parrs Sammlung No. 44 und Anhang No. 17. 
Aurati, et Hiberniae Thesaurarii, in cognitione linguarum Orientalium (etiam abstrusiorum, Armenae, et Aethiopicae) versatissimus, et Arameae seu Syriacae inprimis addictus; a quo, spero, impetrabitur, ut publico bono ista vulgare in animum inducat. Est liber magnus in quarto, dense admodum, angustis marginibus, et minutiori charactere scriptus; titulumque gerit אוצר ראוא [statt i ist fälschlich I gedruckt], Horreum secretorum seu mysteriorum, compositus ante trecentos circiter annos, quantum colligere visus sum ex nonnullis locis." Die gehoffte Herausgabe des ||$_{\mid} ;_{3}$ ol oder eines Extraktes aus demselben durch Loftus ist nicht zu stande gekommen; von der Absicht einer Herausgabe zeugt noch die im Besitze des Trinity College in Dublin befindliche lateinische Uebersetzung von Loftus. Vgl. Catalogus librorum ms. Angliae et Hiberniae II 2, 49, wo unter No. 856 als siebente der Handschriften des Dudley Loftus aufgeführt wird ${ }_{n}$ Expositio Gregorii Maphorini in cunctos libros tam Novi quam Veteris Testamenti per D. Dudl. traducta in Latinum". Nach Bootius und Loftus scheint auch Walton Usshers Handschrift des $\mid\rceil_{1} ; 0$ benutzt oder wenigstens gesehen zu haben; denn er beruft sich Prolegomena $89^{2}$ auf das Zeugnis von Gregorius Syrus in lib[ro] MS. Syriaco ubi Scholia habet in totum V. Test.". Dann aber scheint die Handschrift verschollen zu sein. $\mathrm{Ob}$ sie noch vorhanden ist, und wo sie ist, kann ich nicht sagen. In England sind nach W. Wright in Encycl. Brit. XXII 854 fünf Handschriften des |үї ; Hunt. 1. Brit. Mus. Add. 7186. 21580. 23596. Cambridge, coll. of the S. P. C. K. Die Handschrift der Bodleiana stammt von Huntingdon; die drei Handschriften des British Museum sind erst in diesem Jahrhundert nach England gebracht; uber das der Society for Promoting Christian Knowledge gehörende Exemplar verdanke ich W. Robertson Smith folgende Mitteilung : „The Cambridge Ms of the 
i|i ; 01 is certainly not that of Ussher. It was brought from the East by the late Dr Percy Badger in the mission described in his ,Nestorians and their Rituals" “. Usshers Handschrift ist also entweder verloren gegangen oder harrt, in irgend einer Bibliothek versteckt, ihrer Erlösung aus langjähriger Vergessenheit.

Die Usshersche Handschrift des | |ï ; 01 , über deren Geschichte ich im Vorstehenden das mir Bekannte mitgeteilt habe, war in der Zeit von 1628-1653 die einzige, in England vorhandene Handschrift jenes Werkes. Die Randnoten aus Gregorius in Uss. müssen also aus ihr entnommen sein. Wenn es sich fragt, wer diese Randnòten beigeschrieben hat, so ist es von vornherein am wahrscheinlichsten, dafs der Besitzer beider Handschriften, Ussher, selbst dies gethan hat. Aufser ihm könnte nur jemand in Betracht kommen, welcher beide Handschriften gleichzeitig von ihm entliehen hatte, und in dieser Lage ist, so viel ich weifs, nur A. Bootius gewesen. Es ist aber einerseits unwahrscheinlich, dafs Bootius die Befugnis hatte, so mit den entliehenen Handschriften zu verfahren, und andrerseits läfst sich nicht einsehen, welchen Zweck er bei einem .solchen Verfahren im Auge gehabt haben sollte. Wohl aber lälst sich - und das ist ein weiterer Grund für Usshers Urheberschaft - nachweisen, zu welchem Zwecke Ussher jene Randnoten aus Gregorius in seiner Handschrift des alten Testamentes anbringen konnte. Ich erinnere daran, dafs Constantin l'Empereur Usshers Handschrift des $\mid j ; i$; 301 . Weihnachten 1636 auf Usshers Aufforderung hin an ihn zurückschickte. Der Ueberbringer Boswell wird sie im Anfange des Jahres 1637 an Ussher abgegeben haben. Damals waren in Leyden bei de Dieu noch Usshers syrischer Pentateuch und syrischer Psalter. Jenen hatte er mit dem Briefe 174 vom 9. = 19. Juni 1632 an de Dieu geschickt, diesen gemäfs dem den ${ }_{n}$ Animad- 
versiones in Acta Apostolorum" de Dieu's vorangehenden, an Ussher gerichteten Widmungsschreiben im Jahre 1631. Auch diese beiden Handschriften erbittet Ussher bald darauf, im Juli 1637, von de Dieu zurück (letter 195). Hier giebt er ausdrücklich den Grund an, weshalb er sie jetzt wieder haben müsse. Er beabsichtigt, das syrische alte Testament in Druck zu geben und schickt daher den Träger jenes Briefes (195) nach Leyden, um syrische oder hebräische Typen zu kaufen. „Latorem istorum istuc transmisimus ad coemendos illic Characteres vel Syriacos vel Hebraicos, quibus hîc imprimamus Vetus Testamentum Syriacum." Dem Drucke zu Grunde legen will er seine Handschrift des alten Testamentes, aber daneben will er auch seinen Pentateuch und Psalter benutzen nad tollenda errata alterius nostri exemplaris, in quibus, etiam ubi sunt admodum manifesta, nibil mutare volumus, nisi aliorum codicum auctoritate. " Hiernach ist es wahrscheinlich, dafs er auch seine Handschrift des $|\hat{i}| ; 3,0 \mid$, obwohl er dies nicht ausdrücklich sagt, zu demselben Zwecke zurückgefordert, und dafs er selbst das Wichtigste daraus an den Rand seines alten Testamentes geschrieben hat, um es bei der Redaction des Textes bequem benutzen zu können.

In dem alten Testamente Usshers fehlen die Psalmen. Beim Drucke wollte er dafür offenbar seine Handschrift des Psalters zu Grunde legen. Denn am Rande derselben finden sich, wie Thorndike bemerkt, die Varianten aus der Ausgabe des Thomas Erpenius notiert. Usshers Plan ist somit deutlich $\mathrm{zu}$ erkennen. Er hat sein altes Testament (ohne Psalmen) und seinen Psalter als Druckvorlage benutzen, dieselben aber aus anderen Handschriften und aus dem Commentare des Bar Ebhraya corrigieren wollen.

Dieser Plan ist nicht ausgeführt worden. Zwar spricht Ussher in jenem Briefe (195) die Hoffnung aus, dafs die Arbeit „parvo temporis spatio" werde beendigt sein. Aber sei es, dafs er keine Typen bekam, sei es, dafs ein anderer 
Hinderungsgrund vorlag, seine Hoffnung gieng nicht in Erfüllung. Doch hat er auch den Plan, das alte Testament herauszugeben, nicht fallen lassen. Wie er schon 1637 sich bemüht, aus Leyden andere Bibelhandschriften zur Vergleichung zu bękommen (letter 195), so hat er später seine Handschrift des alten Testamentes nach Paris geschickt, damit sie dort mit einer anderen Handschrift collationiert werde. Walton berichtet darüber in den Prolegomena $89^{2}$ : „Exemplaria vero MSS. quibus usi sumus, duo nobis suppeditavit Reverendissimus Usserius, quorum ... aliud totum continebat Vet. Testamentum exceptis Psalmis . . Hoc vero cum Codice alio Manuscripto contulit vir doctus cum Parisiis agebat, et discrepantias quasdam in margine notavit." Wenn nicht anzunehmen ist, dafs Walton sich unrichtig ausgedrückt und statt ${ }^{\text {vir }}$ doctus" vielmehr „ vir quidam doctus" gemeint hat, so ist auch hier seine Angabe falsch. Denn Ussher selbst ist nie in Paris gewesen, wohl aber seine Handschrift des alten Testamentes, und zwar sicher in der ersten Hälfte des Jahres 1653 (event. auch schon früher). Denn Walton weifs am 18. $=28$. Juli 1653 , wo er Ussher bittet, ihm dieselbe für die Londoner Polyglotte zu leihen, noch nicht, ob sie „be come out of France“ (letter 276) ${ }^{1}$ ). Nicht Ussher selbst hat die Pariser Handschrift collationiert,

1) Obgleich Waltons Ausdruck nicht genau ist, scheint mir doch zweifellos, dafs unter dem in Frankreich befindlichen ${ }_{n}$ Syriack Copy Usshers altes Testament, unter dem schon in Waltons Händen befindlichen „other Manuscript" Usshers Pentateuch zu verstehn ist. Verständlicher werden die ungenauen Ausdrücke, wenn man bedenkt, dafs damals (1653) gerade der Pentateuch bearbeitet wurde. - Daraus, dals Walton von Ussher zuerst den Pentateuch, später das alte Testament bekam, erklärt sich auch, weshalb das in den späteren Büchern als Uss. bezeichnete alte Testament Usshers in den Varianten zum Pentateuche nicht als Uss. 1, sondern als Uss. 2 bezeichnet wird, eine Bezeichnung, welche recht unpraktisch und, wenn man jene Thatsache nicht kennt, kaum zu exklären ist. 
sondern Bootius. Vgl. Thorndike zu Eccli 1, 20 : „Ascriptae erant ad marg. Uss. variae lectiones, ex codice Sebast. Hardy quem contulerat Bootius. Hae ex his notis Hard. aut $\mathrm{H}$. intelligendae." Der Niederländer Arnold de Boot, anglisiert A. Boate, latinisiert A. Bootius, von Profession Arzt, lebte eine Zeit lang in Dublin, wo er, wie schon erwähnt, um 1640 Usshers Handschriften des alten Testamentes und des $|\hat{\eta}| ;$; 0 l benutzte. Später siedelte er nach Paris über, wo er in einer literarischen Fehde gegen (Jo. Morinus und) Lud. Capellus die Unversehrtheit des hebräischen Textes verteidigte. In dieser Fehde baten sowohl Capellus (Parr, letter 262), als de Boot (264) den Erzbischof Ussher, das entscheidende Wort zu sprechen, und dieser liefs sich auch darauf ein, indem er 1652 die „Epistola ad L. Capellum de textus hebraici variantibus lectionibus" (abgedruckt bei Parr 267) veröffentlichte, in welcher er sich im Wesentlichen auf de Boot's Seite stellte; der Erfolg war freilich, wie fast immer in solchen Fällen, dafs er es weder dem de Boot (Parr 271), noch dem Capellus (274) recht machte. De Boot war übrigens ein rechter Heifssporn. Die ihm durch Ussher angebotenen Anmerkungen Eyres zu den Psalmen will er nicht annehmen, wenn Eyre wirklich, wie er vermutet, ein Anhänger des Capellus ist, da er einem solchen nicht zu Danke verpflichtet sein möchte (264). Ueber die Pariser Polyglotte spricht er das Urteil, sie sei „basely defaced with innumerable Faults, and therefore fit for nothing but to be burnt" (288), und da Walton das Verbrechen begangen hat, Morinus und Capellus ehrenvoll zu erwähnen, so ist er auch auf die Londoner Polyglotte recht schlecht zu sprechen (275). Dieser Arnold de Boot, über dessen Persönlichkeit ich etwas Genaueres mitgeteilt habe, da er und seine Schriften jetzt der Vergessenheit anheimgefallen sind, stand von Paris aus in gelehrtem Briefwechsel mit Ussher und sah für ihn mancherlei in Pariser Handschriften nach, 
wie aus den bei Parr abgedruckten Briefen hervorgeht. So schickte ihm Ussher auch seine Handschrift des alten Testamentes, damit er diese mit Pariser Handschriften vergleiche. De Boot hat anscheinend nur den Ecclesiasticus collationiert, denn. nur zu diesem finden sich mit Hard. oder H. bezeichnete Randnoten. Die Handschrift, welche de Boot zur Vergleichung heranzog, gehörte nach Thorndikes Angạbe dem Sébastien Hardy. Genaueres über dieselbe habe ich nicht ermitteln können. Doch glaube ich, dafs auch hier ein Irrtum vorliegt, und dafs die Handschrift nicht dem Sébastien Hardy, welcher zwar literarisch thätig war, aber sich, soweit mir bekannt ist, nicht mit den orientalischen Sprachen beschäftigte (s. über ihn B. Hauréau, histoire littéraire du Maine, nouvelle édition VI 72), gehört hat, sondern dessen Sohne, dem mit Descartes befreundeten Juristen, Mathematiker und Orientalisten Claude Hardy, königlichem Rate in Paris (conseiller au Châtelet), über welchen Colomies, Gallia orientalis $165 \mathrm{f}$. $259 \mathrm{f}$. zu vergleichen ist.

Hieraus ergiebt sich, dafs Ussher sich noch 1653 mit dem Gedanken getragen hat, das syrische alte Testament herauszugeben. $\mathrm{Ob}$ er, als nun die Londoner Polyglotte gedruckt, und dafür auch seine Handschriften verglichen wurden, seinen Plan aufgegeben hat, oder ob sein 1656 erfolgender Tod der Ausführung desselben zuvorgekommen ist, mufs dahingestellt bleiben.

In neuerer Zeit hat Samuel Lee unter anderem auch Uss. für seine Ausgabe des alten Testamentes eingesehen (Classical Journal vol. 23, 245-249), doch scheint er seine Handschriftenstudien nur sehr flüchtig betrieben zu haben. Wesentlicher Nutzen für seine Ausgabe ist jedenfalls nicht daraus geflossen, da er im Ganzen blols einen Abdruck der Londoner Polyglotte bietet. Varianten durften der Ausgabe, da sie .im Auftrage der Bibelgesellschaft erschien, nicht beigegeben werden. Lee hat versprochen seinVarianten- 
verzeichnis separat erscheinen zu lassen, doch ist dies Versprechen, so viel ich weifs, nie erfullt worden.

Uss. ist trotz der daran gewand ten Bemühungen Usshers, Thorndikes und Lees nur sehr unvollständig bekannt. Für eine künftige Ausgabe der Peschita wird Uss. vollständig verglichen werden müssen.

\section{Nachtrag.}

Als ich diese Arbeit anfertigte, besafs, wie ich S. 193 Anm. 2 bemerkt habe, die Göttinger Bibliothek die Gesamtausgabe der Werke Usshers noch nicht. Jetzt hat aber Herr Oberbibliothekar Prof. Dziatzko die Güte gehabt, diese Ausgabe für die Göttinger Bibliothek anzuschaffen, und so sehe ich mich in der Lage, dieselbe noch nachträglich benutzen zu können.

Da Elrington von Parr in der Anordnung und Zählung der Briefe abweicht, gebe ich eine Liste der von mir citierten Nummern Parrs und der ihnen entsprechenden Elringtons :

\begin{tabular}{cc|cc|cc} 
Parr & Elrington & Parr & Elrington & Parr & Elrington \\
44 & 46 & 177 & 192 & 271 & 299 \\
69 & 71 & 180 & 196 & 274 & 302 \\
81 & 102 & 182 & 198 & 275 & 303 \\
83 & 90 & 192 & 206 & 276 & 304 \\
99 & 110 & 195 & 210 & 288 & 318 \\
120 & 125 & 197 & 212 & 305 & 220 \\
122 & 127 & 262 & 290 & 306 & 226 \\
174 & 184 & 264 & 291 & Anhang & 17 fehlt \\
175 & 188 & 267 & $295(=$ vol. 7, & & \\
& & & p. 589-609) & &
\end{tabular}

Aus den bei Parr noch nicht gedruckten Briefen der Ausgabe Elringtons ist nachzutragen

1) zu S. 194 Anm. 2 : Letter 388 von Thomas Davies, 13. März $1626=23$. Marz $1627:{ }_{n}$ The Psalter in the Syriac, according to the Greek, I cannot find as yet".

2) zu S. 203, Anm. 1 : Der Brief, mit welchem de Dieu den Katalog der Leydener Handschriften an Ussher geschickt hat, ist erhalten bei Elrington No. 425. Hier ist or datiert ${ } 16$ Decemb. $1635^{\star}$. Dies ist aber offenbar ein Fehler, da der Brief den am 14. = 24. Sept. 1636 geschriebenen Brief Usshers Parr $188=$ Elrington 205 beantwortet. Es muls ${ }_{n} 16$. Dec. $1636^{\alpha}$ heifsen. Hierdurch wird zugleich das Datum ${ }_{n}$ Weihnacht $1636^{4}$ für l'Empereurs Brief bestätigt.

3) zu S. 205, Z. 3-6 : Usshers Handschrift des Horreum mysteriorum ist schon zu seinen Lebzeiten - auf welche Weise, ist ungewils - verloren gegangen, wie aus A. de Boots ${ }_{n}$ Paris, 15/25 Jan. 1652/3 datiertem Briefe Elrington 449 hervorgeht : ${ }_{n} \mathrm{I}$ am sorry ... for what you tell me of Gregorius Scholiastes, the loss whereof doth most sensibly grieve me". 